\title{
Thermodynamics and complex dielectric permittivity of mixed crystals of the $\mathrm{Rb}_{1-x}\left(\mathrm{NH}_{4}\right)_{x} \mathrm{H}_{2} \mathrm{PO}_{4}$ type
}

\author{
R.R.Levitsky ${ }^{1}$, S.I.Sorokov ${ }^{1}$, J.Stankowski ${ }^{2}$, Z.Trybula ${ }^{2}$, A.S.Vdovych ${ }^{1}$ \\ ${ }^{1}$ Institute for Condensed Matter Physics of the National Academy of Sciences of Ukraine, \\ 1 Svientsitskii Str, 79011 Lviv, Ukraine \\ 2 Institute of Molecular Physics, Polish Academy of Sciences, Smoluchowskiego 17, 60-179 Poznan, Poland
}

Received June 10, 2008

\begin{abstract}
We propose a pseudospin model for proton glasses of the $\mathrm{Rb}_{1-x}\left(\mathrm{NH}_{4}\right)_{x} \mathrm{H}_{2} \mathrm{PO}_{4}\left(\mathrm{Rb}_{1-x}\left(\mathrm{ND}_{4}\right)_{x} \mathrm{D}_{2} \mathrm{PO}_{4}\right)$ type, which takes into account the energy levels of hydrogens (deuterons) around the $\mathrm{PO}_{4}$ group, long-range interactions between the hydrogen bonds, and an internal random deformational field. Within the framework of a cluster approximation and a mean field approximation over the long-range interactions, we derive a system of equations for the state parameters for the regions which are in the ferroelectric and antiferroelectric states, as well as in the proton glass state. Within the Glauber dynamics approach, we obtain a system of equations for the frequency-dependent linear responses of polarization and the proton glass order parameter. We obtain a qualitative description of the temperature behavior of dielectric permittivities of the $\mathrm{K}_{1-x}\left(\mathrm{ND}_{4}\right)_{x} \mathrm{D}_{2} \mathrm{PO}_{4}$ and $\mathrm{Rb}_{1-x}\left(\mathrm{NH}_{4}\right)_{x} \mathrm{H}_{2} \mathrm{AsO}_{4}$ compounds at different frequencies. The origin of low-temperature peak in the imaginary part of dielectric permittivity in proton glasses is discussed.
\end{abstract}

Key words: proton glass, cluster approximation, Glauber dynamics

PACS: $75.10 . \mathrm{Hk}, 75.10 . \mathrm{Nr}, 77.22 . \mathrm{Ch}, 77.22 . \mathrm{Gm}, 77.84 . \mathrm{Fa}$

\section{Introduction}

A notion of structural glasses (now called proton glasses) was introduced by E. Courtens in [1] during investigations of the temperature behavior of transverse and longitudinal permittivities (at $\nu=\omega / 2 \pi=1 \mathrm{kHz})$ of the $\mathrm{Rb}_{1-x}\left(\mathrm{NH}_{4}\right)_{x} \mathrm{H}_{2} \mathrm{PO}_{4}$ system $\left(T_{\mathrm{c}}=147.8 \mathrm{~K}\right.$ for $\mathrm{RbH}_{2} \mathrm{PO}_{4}, T_{\mathrm{N}}=148 \mathrm{~K}$ for $\left.\mathrm{NH}_{4} \mathrm{H}_{2} \mathrm{PO}_{4}\right)$ at $x \leqslant 0.35$. At $x \geqslant 0.2$ the studies of the real part of dielectric permittivity $\varepsilon_{11}^{\prime}(\omega, T)$ revealed that with temperature lowering, the system first undergoes a transition to a mixed state at a certain temperature $T_{f}(\omega)$ (a deviation from the Curie-Weiss law is observed) and then, after reaching a plateau-like maximum (at $\left.T_{\max }(\omega)\right)$, to a structural glass state at temperature $T_{g}(\omega)$ (when $\varepsilon_{11}^{\prime}(\omega, T)$ starts to decrease). In [2] by analyzing an expression for dielectric susceptibility $\chi_{33}(\omega, T)$ a rectangular approximation for distribution function of relaxation times $g(\tau, T)$ was proposed. A maximal relaxation time $\tau_{m}$ at $x=0.35$ is well described by the Vogel-Fulcher law $\left(T_{g}(\omega \rightarrow 0) \rightarrow T_{0}\right)$

$$
\chi_{33}(\omega, T)=\chi_{0}(T) \cdot \int_{0}^{\infty} \mathrm{d} \ln \tau \cdot \frac{g(\tau, T)}{1-\mathrm{i} \omega \tau}, \quad \tau_{m}=\tau_{0} \cdot \exp \left(\frac{E_{\mathrm{c}}}{T-T_{0}}\right), \quad T_{0}=8.74 \mathrm{~K} .
$$

Later, on the basis of dielectric [2-8], optical [9], and X-ray scattering $[6,7,10]$ measurements a phase diagram of the $\mathrm{Rb}_{1-x}\left(\mathrm{NH}_{4}\right)_{x} \mathrm{H}_{2} \mathrm{PO}_{4}$ was constructed, in which the concentration range of about $0.20<x<0.75$ corresponds to the proton glass state. Ferroelectric and antiferroelectric phase transitions were detected at lower and higher concentrations, respectively.

In [11] it was shown for $\mathrm{Rb}_{0.53}\left(\mathrm{NH}_{4}\right)_{0.47} \mathrm{H}_{2} \mathrm{PO}_{4}$ that the spectrum of distribution function $g(\tau, T)$ between $55 \mathrm{~K}$ and $35 \mathrm{~K}$ consists of two wide lines, and with temperature decreasing, a fast crossover of intensity from one band to the other takes place (a peak is observed at $\tau_{\max } \sim 10^{-5} \mathrm{~s}$ 
at $T=55 \mathrm{~K}$ and at $\tau_{\max } \sim 10^{2} \mathrm{~s}$ for $\left.T=35 \mathrm{~K}\right)$. To explain this, the authors use a model of dynamically correlated domains (DCD), for which distribution functions of the domain number and of relaxation frequencies on the domain size are introduced. At freezing temperature some of the domains form an infinite percolation cluster, and the system goes to a non-ergodic state.

In [12], the studies of relaxation times of the ${ }^{87} \mathrm{Rb} \mathrm{NMR}$ spectra for $\mathrm{Rb}_{0.58}\left(\mathrm{ND}_{4}\right)_{0.42} \mathrm{D}_{2} \mathrm{PO}_{4}$ and $\mathrm{Rb}_{0.5}\left(\mathrm{NH}_{4}\right)_{0.5} \mathrm{H}_{2} \mathrm{PO}_{4}$ revealed that at low temperatures $T<25 \mathrm{~K}$ for $\mathrm{Rb}_{0.5}\left(\mathrm{NH}_{4}\right)_{0.5} \mathrm{H}_{2} \mathrm{PO}_{4}$ one should take into account the relaxation time related to phonon-activated tunneling $\tau_{\Gamma}$. Plateau of ${ }^{87} \mathrm{Rb}$ relaxation time in a pure $\mathrm{RbH}_{2} \mathrm{PO}_{4}$, which at these temperatures is in the ferroelectric ordering state, turned out to be $10^{4}$ times higher than for the proton glass $\mathrm{Rb}_{0.5}\left(\mathrm{NH}_{4}\right)_{0.5} \mathrm{H}_{2} \mathrm{PO}_{4}$. This indicates strong differences in the relaxation mechanisms in pure systems with hydrogen bonds and in their mixtures.

Various mixed hydrogen bonded ferroelectrics of this type have been studied: $\mathrm{K}_{1-x}\left(\mathrm{NH}_{4}\right)_{x} \mathrm{H}_{2} \mathrm{PO}_{4}$ $\left(T_{\mathrm{c}} \approx 122.5 \mathrm{~K}\right.$ for $\mathrm{KH}_{2} \mathrm{PO}_{4}, T_{\mathrm{N}} \approx 148 \mathrm{~K}$ for $\left.\mathrm{NH}_{4} \mathrm{H}_{2} \mathrm{PO}_{4}\right), \mathrm{Rb}_{1-x}\left(\mathrm{NH}_{4}\right)_{x} \mathrm{H}_{2} \mathrm{AsO}_{4}\left(T_{\mathrm{c}} \approx 110 \mathrm{~K}\right.$ for $\mathrm{RbH}_{2} \mathrm{AsO}_{4}, T_{\mathrm{N}} \approx 216 \mathrm{~K}$ for $\left.\mathrm{NH}_{4} \mathrm{H}_{2} \mathrm{AsO}_{4}\right)$ as well as their deuterated analogues $\mathrm{K}_{1-x}\left(\mathrm{ND}_{4}\right)_{x} \mathrm{D}_{2} \mathrm{PO}_{4}$ $\left(T_{\mathrm{c}} \approx 220 \mathrm{~K}\right.$ for $\mathrm{KD}_{2} \mathrm{PO}_{4}, T_{\mathrm{N}} \approx 242 \mathrm{~K}$ for $\left.\mathrm{ND}_{4} \mathrm{D}_{2} \mathrm{PO}_{4}\right), \mathrm{Rb}_{1-x}\left(\mathrm{ND}_{4}\right)_{x} \mathrm{D}_{2} \mathrm{AsO}_{4}\left(T_{\mathrm{c}} \approx 174 \mathrm{~K}\right.$ for $\mathrm{RbD}_{2} \mathrm{AsO}_{4}, T_{\mathrm{N}} \approx 304 \mathrm{~K}$ for $\left.\mathrm{ND}_{4} \mathrm{D}_{2} \mathrm{AsO}_{4}\right)$.

In this work we shall explore dielectric properties of $\mathrm{Rb}_{1-x}\left(\mathrm{ND}_{4}\right)_{x} \mathrm{D}_{2} \mathrm{PO}_{4}$ and $\mathrm{Rb}_{1-x}\left(\mathrm{NH}_{4}\right)_{x} \mathrm{H}_{2} \mathrm{AsO}_{4}$ ferroelectrics. In the first one, a rather high value of $T_{g} \sim 60 \mathrm{~K}$ is observed; also in deuterated compounds one can neglect tunneling effects. In contrast to a symmetric behaviour over $x$ phase diagram of $\mathrm{Rb}_{1-x}\left(\mathrm{ND}_{4}\right)_{x} \mathrm{D}_{2} \mathrm{PO}_{4}$, the diagram of a $\mathrm{Rb}_{1-x}\left(\mathrm{NH}_{4}\right)_{x} \mathrm{H}_{2} \mathrm{AsO}_{4}$ mixture $\left(T_{g} \sim 30 \mathrm{~K}\right)$ is strongly asymmetric. For $\mathrm{Rb}_{1-x}\left(\mathrm{NH}_{4}\right)_{x} \mathrm{H}_{2} \mathrm{AsO}_{4}$ we can find out how important it is to take tunneling into account in calculations of dielectric characteristics.

Investigations of the state of proton glasses in $\mathrm{Rb}_{1-x}\left(\mathrm{ND}_{4}\right)_{x} \mathrm{D}_{2} \mathrm{PO}_{4}$ started in [13] for $x=0.55$ and [14] for $x=0.44$ using the NMR method. It was shown [14] that a satisfactory description of temperature dependence of Edwards-Anderson parameter $q_{\mathrm{EA}}(T)$ is possible within the framework of a model which takes into account fluctuations of interactions between hydrogen bonds and chaotic deformational field (the random-field random-bond Sherrington-Kirkpatrick Ising model).

In [15] the Edwards-Anderson $q_{\mathrm{EA}}(T)$ parameter was studied using the NMR method for $x=$ $0.22,0.44,0.78$ at different orientations of external magnetic field with respect to the $c$ axis of the crystal.

Temperature and frequency curves of transverse $\varepsilon_{11}^{\prime}(\omega, T), \varepsilon_{11}^{\prime \prime}(\omega, T)$ and longitudinal $\varepsilon_{33}^{\prime}(\omega, T)$, $\varepsilon_{33}^{\prime \prime}(\omega, T)$ permittivities of $\mathrm{Rb}_{1-x}\left(\left[\mathrm{~N}\left(\mathrm{H}_{1-y} \mathrm{D}_{y}\right)_{4}\right]_{x}\left(\mathrm{H}_{1-y} \mathrm{D}_{y}\right)_{2} \mathrm{PO}_{4}(0.25 \leqslant x \leqslant 0.75,0 \leqslant y \leqslant 1.0)\right.$ were measured at $4.2 \mathrm{~K} \leqslant T \leqslant 300 \mathrm{~K}, 1 \mathrm{kHz} \leqslant \nu \leqslant 1 \mathrm{GHz}$ in [16]. It was shown that the experimental data for the permittivities are well described by the distribution function of relaxation times $g(\tau, T)$ calculated from phenomenological relation between $\tau$ and Gaussian distribution $w\left(E, \bar{E}, \sigma_{\mathrm{E}}\right)$ of the activation energy $E$

$$
\tau=\tau_{0} \exp \left[E /\left(T-T_{0}\right)\right], \quad w\left(E, \bar{E}, \sigma_{\mathrm{E}}\right)=\frac{1}{\sqrt{2 \pi} \sigma_{\mathrm{E}}} \exp \left\{-\frac{1}{2} \frac{(E-\bar{E})^{2}}{\sigma_{\mathrm{E}}^{2}}\right\} .
$$

In [17] a phase diagram was presented; it was also shown that the system dynamics on the proton glass state $(x=0.5,0.7)$ is thermally activated, in contrast to a soft mode behavior in the regions with predominantly ferroelectric and antiferroelectric orderings.

In [18] the transverse static $\varepsilon_{11}(T)$ and low-frequency dynamic $\varepsilon_{11}^{\prime}(\omega, T)$ permittivities of the $\mathrm{Rb}_{1-x}\left(\mathrm{ND}_{4}\right)_{x} \mathrm{D}_{2} \mathrm{PO}_{4}$ mixture at different compositions $(x=0.15-0.70)$ were measured. For the distribution function of relaxation times $g(\tau, T)$ a linear approximation with respect to the $z=\ln \omega_{a} \tau$ parameter was proposed. A maximal relaxation time $\tau_{m}$ diverges with temperature according to the Vogel-Fulcher law. Interpretation of separation lines $T_{\mathrm{c}}(x)$ between EPG (Ergodic Proton Glass) and EF (Ergodic Ferromagnetic) phases and $T_{\mathrm{N}}(x)$ between EPG and EAF (Ergodic Antiferromagnetic) phases, as well as the freezing lines $T_{0}(x) \approx 33 \mathrm{~K} \pm 0.5 \mathrm{~K}$ between EPG and NPG (Nonergodic Proton Glass) were performed using a random-field random-bond Ising model. Here the freezing line $T_{0}=T_{0}(x)$ is interpreted as an Almeida-Thoulesse instability line, below which the replica-symmetric (RS) solutions are incorrect. This work did not contain a theoretical description of $\varepsilon_{11}(T), \varepsilon_{11}^{\prime}(\omega, T)$ temperature curves. 
In [19] the permittivities $\varepsilon_{11}^{\prime}(\omega, T), \varepsilon_{11}^{\prime \prime}(\omega, T)$ of $\mathrm{Rb}_{0.6}\left(\mathrm{ND}_{4}\right)_{0.4} \mathrm{D}_{2} \mathrm{PO}_{4}$ were measured in a wide frequency range $(\nu=1 \mathrm{mHz}-1 \mathrm{MHz})$ at temperatures $T \in[25 \mathrm{~K}-100 \mathrm{~K}]$. Using the inverse integral transformation, a distribution function $g(\tau, T)$ was constructed, and three first moments $\langle\tau\rangle$, $\left\langle\tau^{2}\right\rangle^{\text {cum }},\left\langle\tau^{3}\right\rangle^{\text {cum }}$ were calculated. The average value of $\langle\tau\rangle$ is a satisfactory approximation by the Vogel-Fulcher law with $T_{0}(0.4) \approx 16.5 \mathrm{~K} \pm 1.2 \mathrm{~K}$.

In [20] the transverse $\varepsilon_{11}^{\prime}(\omega, T), \varepsilon_{11}^{\prime \prime}(\omega, T)$ and longitudinal $\varepsilon_{33}^{\prime}(\omega, T), \varepsilon_{33}^{\prime \prime}(\omega, T)$ permittivities of $\mathrm{Rb}_{0.6}\left(\mathrm{ND}_{4}\right)_{0.4} \mathrm{D}_{2} \mathrm{PO}_{4}$ were compared.

In [21], the $\mathrm{NMR}$ studies of $\mathrm{Rb}_{0.22}\left(\mathrm{ND}_{4}\right)_{0.78} \mathrm{D}_{2} \mathrm{PO}_{4}$ showed that proton glass state is formed due to gradual freezing of deuterons on hydrogen bonds. It also leads to deformation and rotation of $\mathrm{ND}_{4}$ groups.

In [22], the dielectric properties of $\mathrm{Rb}_{0.5}\left(\mathrm{ND}_{4}\right)_{0.5} \mathrm{D}_{2} \mathrm{PO}_{4}$ as well as microwave and millimeter frequency range were explored. The distribution function of relaxation times $g(\tau, T)$ at $120 \mathrm{~K}<$ $T<170 \mathrm{~K}$ calculated from the experimental data is well described by the theoretical curve calculated using a Gaussian averaging of phenomelogical relation for $\tau$ with Gaussian distribution for activation energy $E$ and parameter of the potential well asymmetry.

The most complete experimental data for $\mathrm{Rb}_{1-x}\left(\mathrm{NH}_{4}\right)_{x} \mathrm{H}_{2} \mathrm{AsO}_{4}$ for the dielectric measurements in a wide frequency range at low temperatures can be found in [23-29].

In [23], a temperature dependence $(T=5 \mathrm{~K}-300 \mathrm{~K})$ of longitudinal permittivity $\varepsilon_{33}^{\prime}(\omega, T)$, $\varepsilon_{33}^{\prime \prime}(\omega, T)$ of $\mathrm{Rb}_{1-x}\left(\mathrm{NH}_{4}\right)_{x} \mathrm{H}_{2} \mathrm{AsO}_{4}$ in the millimeter frequency range is measured, and the phase diagram is constructed. In [24], the transverse $\varepsilon_{11}^{\prime}(\omega, T), \varepsilon_{11}^{\prime \prime}(\omega, T)$ and longitudinal $\varepsilon_{33}^{\prime}(\omega, T)$, $\varepsilon_{33}^{\prime \prime}(\omega, T)$ permittivities of $\mathrm{Rb}_{0.65}\left(\mathrm{NH}_{4}\right)_{0.35} \mathrm{H}_{2} \mathrm{AsO}_{4}$ are explored in wide temperature $(T=5 \mathrm{~K}-$ $300 \mathrm{~K})$ and frequency $(\nu=1 \mathrm{~Hz}-30 \mathrm{kHz})$ ranges.

In [25], by measuring transverse dielectric permittivity of $\mathrm{Rb}_{1-x}\left(\mathrm{NH}_{4}\right)_{x} \mathrm{H}_{2} \mathrm{AsO}_{4}$, the occurrence of proton glass state was revealed at low temperatures in the regions of ferroelectric phase (small $x$ ) and antiferroelectric phase (large $x$ ). Later [27], the proton glass state was detected even at $x=0.01$.

Let us now briefly review some theoretical works on the thermodynamic and dielectric properties of the $\mathrm{Rb}_{1-x}\left(\mathrm{NH}_{4}\right)_{x} \mathrm{H}_{2} \mathrm{PO}_{4}$ type proton glasses.

To the best of our knowledge, the first theory for the $\mathrm{Rb}_{1-x}\left(\mathrm{NH}_{4}\right)_{x} \mathrm{H}_{2} \mathrm{PO}_{4}$ mixture was proposed in [30]. A pseudospin Hamiltonian was used to describe the energy levels of hydrogens around a $\mathrm{PO}_{4}$ group; a cluster approximation was used to calculate the transition lines $T_{\mathrm{c}}(x), T_{\mathrm{N}}(x)$. A qualitative description of the phase diagram was obtained.

Later, the cluster approach was used in [31,32]. In [31] to describe the $\mathrm{Rb}_{1-x}\left(\mathrm{NH}_{4}\right)_{x} \mathrm{H}_{2} \mathrm{PO}_{4}$ mixtures, a pseudospin model was employed which took into account a configurational energy of proton bonds cluster around $\mathrm{PO}_{4}$ group, as well as the long-range interaction. The free energy was presented as a sum of the energies of three phases with the probabilities $p_{+}(x)$ for ferroelectric phase, $p_{-}(x)$ for antiferroelectric phase, and $p_{0}(x)$ for neutral phase. By analyzing the coefficients of free energy expansion in the $\left\langle S_{1}\right\rangle+\left\langle S_{3}\right\rangle ;\left\langle S_{1}\right\rangle-\left\langle S_{3}\right\rangle$ parameter, the regions of ferroelectric $(0<x<0.2$ at $T=0)$ and antiferroelectric $(0.75<x<1$ at $T=0)$ phases were found, which were close to experimental data. This model was also used [32] to describe the phase diagram in the proton glass region $(0.2<x<0.75$ at $T=0)$. A RS approximation was employed for averaging the system free energy; an explicit expression for $T_{g}(x)$ was obtained. Thus, in [31,32] not a single approach for description of all states of this type of compounds was developed.

Experimental data are most often interpreted using the random-field random-bond Ising model (also with transverse field) within the framework of RS approach [33]. This model does not take into account a real structure of the crystals. A Gaussian distribution of random interactions with infinite correlation range $\left(\left\langle J_{i j}^{2}\right\rangle_{\mathrm{c}}=x(1-x) \cdot \operatorname{const}(i-j)\right)$, Gaussian chaotic deformational field $h_{i}\left(\left\langle h_{i}\right\rangle=0,\left\langle h_{i}^{2}\right\rangle \sim x(1-x)\right)$, and the RS-approximation are used. It is shown that $T_{g}$ (protonglass transition temperature) exists at $\left\langle h_{i}^{2}\right\rangle_{\mathrm{c}}=0$ only and corresponds to the peak on a temperature curve of $\chi(T)$. Internal chaotic field $\left(\left\langle h_{i}^{2}\right\rangle_{\mathrm{c}} \neq 0\right)$ gives rise to the occurrence of the proton glass state at a rather high temperature above $T_{g}(q>0, \quad q \underset{T \rightarrow \infty}{\longrightarrow} 0)$ and smears out the peak of the $\chi(T)$ temperature curve.

This model was successfully used in [34] to describe a $\mathrm{Tl}^{2+}$ EPR spectrum in 
$\mathrm{Rb}_{0.52}\left(\mathrm{NH}_{4}\right)_{0.48} \mathrm{H}_{2} \mathrm{PO}_{4}$. The Edwards-Anderson parameter $q$ is proportional to the second moment of the EPR distribution function and tends to zero at $T \gg 200 \mathrm{~K}$. This confirms the occurrence of significant static random fields in $\mathrm{Rb}_{1-x}\left(\mathrm{NH}_{4}\right)_{x} \mathrm{H}_{2} \mathrm{PO}_{4}$ compounds. In [35], the peculiarities of low-temperature (slow motion) dynamics are taken into account within the Glauber approach. The observed shape of the

$$
I\left(\omega, \tau_{0}\right)=\int_{-1}^{1} \mathrm{~d} p \cdot W(p) \cdot I\left(\omega, \tau_{0}, p\right)
$$

line contains an integral with the distribution function $W(p)$ of local polarization $\left(\tau_{0}\right.$ is the relaxation time related to the two-well potential). The theoretical curves satisfactorily describe the shape of the line (a single peak at high temperatures and two peaks at low temperatures) in a wide temperature range $(T=[10 \mathrm{~K}, 150 \mathrm{~K}])$.

In [36] the random-field random-bond Ising model with phonon thermostat is used to calculate dielectric permittivity of $\mathrm{Rb}_{1-x}\left(\mathrm{ND}_{4}\right)_{x} \mathrm{D}_{2} \mathrm{PO}_{4}$ and $\mathrm{Rb}_{1-x}\left(\mathrm{ND}_{4}\right)_{x} \mathrm{D}_{2} \mathrm{AsO}_{4}$. The theory yields Debye type relaxation, with the relaxation time considered as a phenomenological parameter taken according to the Arrhenius law. A quantitative comparison is performed for the temperature dependence of $\varepsilon^{\prime \prime}(\omega)$ peak; the entire temperature curves of permittivity $\varepsilon^{\prime}(\omega, T), \varepsilon^{\prime \prime}(\omega, T)$ are not described.

In $[37,38]$ a model Hamiltonian is used, the parts of which describe ferroelectric ordering along the $Z$ axis ( $S^{z}$ components of a classical spin) and antiferroelectric ordering ( $S^{x}$ component). Instead of the generally accepted Edwards-Anderson parameter $q_{\mathrm{EA}}$, single-site correlators $\left\langle S_{i 1}^{z} S_{i 2}^{z}\right\rangle$, $\left\langle S_{i 1}^{x} S_{i 2}^{x}\right\rangle$ for sublattices 1, 2 are used as an order parameter for the proton glass phase. The phase diagram for $\mathrm{Rb}_{n}\left(\mathrm{NH}_{4}\right)_{1-n} \mathrm{H}_{2} \mathrm{AsO}_{4}$ constructed within $\mathrm{RS}$ approximation qualitatively correctly describes some experimental phase boundaries.

Hence, a theoretical description of thermodynamic and dielectric properties of the hydrogen bonded compounds of $\mathrm{Rb}_{1-x}\left(\mathrm{NH}_{4}\right)_{x} \mathrm{H}_{2} \mathrm{PO}_{4}$ type, which can undergo a transition into the proton glass state, that takes into account structural peculiarities and different types of interactions, is still an important and unresolved problem of statistical physics. Especially important is a microscopic description of relaxational properties of this type of mixtures. It is interesting to describe temperature curves of real and imaginary parts of longitudinal and transverse dielectric permittivities at different frequencies, in particular, the low-temperature portion of the imaginary part of the permittivity, whose peak indicates a transition to a non-ergodic state.

In [39], on the basis of the cluster expansion method, a single approach is suggested to a theory of proton ordering in ferroelectrics and antiferroelectrics of the orthophosphate type where the configuration short-range, dipole long-range interactions are taken into account. In [40,41] a theory of static properties of model proton glasses with an arbitrary range of competing interactions was proposed. Thermodynamic properties of the model with essential short-range and weak long-range interactions were explored within the two-particle cluster approximation. In the present paper, the approaches developed in [39-41] are used to describe thermodynamic and dielectric properties of hydrogen bonded $\mathrm{K}_{1-x}\left(\mathrm{ND}_{4}\right)_{x} \mathrm{D}_{2} \mathrm{PO}_{4}$ and $\mathrm{Rb}_{1-x}\left(\mathrm{NH}_{4}\right)_{x} \mathrm{H}_{2} \mathrm{AsO}_{4}$ compounds.

\section{Thermodynamic properties of the compounds of $\mathrm{Rb}_{1-x}\left(\mathrm{NH}_{4}\right)_{x} \mathrm{H}_{2} \mathrm{PO}_{4}$ type}

In order to describe possible configurations in a mixed $\mathrm{Rb}_{1-x}\left(\mathrm{NH}_{4}\right)_{x} \mathrm{H}_{2} \mathrm{PO}_{4}$ crystal, let us first consider a structure of pure RDP- $\mathrm{RbH}_{2} \mathrm{PO}_{4}$ and $\mathrm{ADP}-\mathrm{NH}_{4} \mathrm{H}_{2} \mathrm{PO}_{4}$ crystals (in figure 1 a unit cell of the isomorphic to RDP for the $\mathrm{KDP}-\mathrm{KH}_{2} \mathrm{PO}_{4}$ crystal is shown).

A primitive cell of $\mathrm{RDP}-\mathrm{RbH}_{2} \mathrm{PO}_{4}$ contains one tetrahedron of the " $A$ " type and one tetrahedron of the " $B$ " type, two Rb atoms and four protons on four hydrogen bonds. In the ferroelectric phase, the total dipole moment of the cell, created by displacements of different ions and by deformation of $\mathrm{PO}_{4}$ group, is directed along the $c$ axis. A triggering mechanism for ionic displacements is proton ordering (their positions are described by spin variables $S_{f}= \pm 1, f=1-4$ ) on double potential wells of the hydrogen bonds. The bond dipole moments lie almost within the $a b$ plane; 
the total dipole moment of the protons for RDP is zero

$$
\vec{\mu}_{1 \alpha}=\left(\mu_{\alpha}^{x}, 0,0\right), \quad \vec{\mu}_{3 \alpha}=\left(-\mu_{\alpha}^{x}, 0,0\right), \quad \vec{\mu}_{2 \alpha}=\left(0,-\mu_{\alpha}^{y}, 0\right), \quad \vec{\mu}_{4 \alpha}=\left(0, \mu_{\alpha}^{y}, 0\right) .
$$

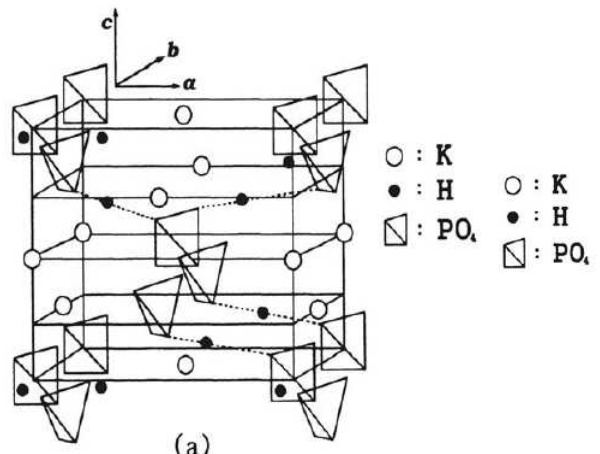

(a)

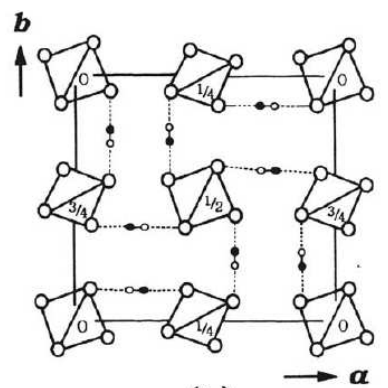

(b)

Figure 1. A unit cell (four formula units) for the $\mathrm{KDP}-\mathrm{KH}_{2} \mathrm{PO}_{4}$ crystal.

It is well known that in order to describe the thermodynamic characteristics and dielectric properties (in a certain frequency range) of these crystals within the pseudospin-phonon model, the ionic variables can be excluded in the static approximation. The system description is then performed within the framework of a pseudospin model with renormalized moments of hydrogen bonds $\vec{d}_{f, \alpha}(\alpha=+$ for RDP, $\alpha=-$ for ADP $)$

$$
\begin{gathered}
\vec{d}_{1 \alpha}=\left(d_{\alpha}^{x}, 0, d_{\alpha}^{z}\right), \quad \vec{d}_{3 \alpha}=\left(-d_{\alpha}^{x}, 0, d_{\alpha}^{z}\right), \quad \vec{d}_{2 \alpha}=\left(0,-d_{\alpha}^{y}, d_{\alpha}^{z}\right), \quad \vec{d}_{4 \alpha}=\left(0, d_{\alpha}^{y}, d_{\alpha}^{z}\right) ; \\
\vec{P}_{\alpha}^{A(B)}=\sum_{f \in A(B)} \vec{d}_{f, \alpha} \eta_{f, \alpha}^{A(B)} ; \quad \eta_{f, \alpha}^{A(B)}=\left\langle S_{f, \alpha}\right\rangle^{A(B)} .
\end{gathered}
$$

Here, we introduced an effective dipole moment of a tetrahedron $\vec{P}_{\alpha} ;\langle\ldots\rangle$ stand for a thermodynamic average; summation $f=A(B)$ is carried out over the bonds, on which the protons order close to the given tetrahedron $A(B)$. For RDP, the tetraderon polarization can have two opposite values along the $c$ axis, when two protons are ordered close to the upper edge of the tetrahedron $\left(\eta_{f}=\eta\right)$ and close to the lower one $\left(\eta_{f}=-\eta\right)$

$$
\eta_{f}=\eta \Rightarrow \vec{P}_{+}^{A(B)}=\left(0,0,2 d_{+}^{z} \eta\right), \quad \eta_{f}=-\eta \Rightarrow \vec{P}_{+}^{A(B)}=\left(0,0,-2 d_{+}^{z} \eta\right) .
$$

For $\mathrm{ADP}-\mathrm{NH}_{4} \mathrm{H}_{2} \mathrm{PO}_{4}$, the primitive cell is twice as large as for RDP, and in addition to " $A$ ", " $B$ " tetrahedra, it contains " $A$ ", " $B$ " tetrahedra. Since their polarizations are opposite to those of " $A$ ", " $B$ ", the total cell polarization is zero:

$$
\begin{array}{ll}
-\eta_{1,-}^{A}=-\eta_{2,-}^{A}=\eta_{3,-}^{A}=\eta_{4,-}^{A}=\eta ; & \vec{P}_{-}^{A}=-\vec{P}_{-}^{A^{\prime}}=\left(-d_{-}^{x} \eta ;+d_{-}^{y} \eta ; 0\right), \\
-\eta_{1,-}^{B}=\eta_{2,-}^{B}=\eta_{3,-}^{B}=-\eta_{4,-}^{B}=\eta ; & \vec{P}_{-}^{B}=-\vec{P}_{-}^{B^{\prime}}=\left(-d_{-}^{x} \eta,-d_{-}^{y} \eta, 0\right) .
\end{array}
$$

For an $\mathrm{ADP}-\mathrm{NH}_{4} \mathrm{H}_{2} \mathrm{PO}_{4}$ crystal the change of sign of $\eta_{f,-}^{A(B)}$ at transition to the " $A^{\prime \prime}$, " $B^{\prime \prime}$ " tetrahedra can be taken into account as (here $\vec{n}$ is the RDP primitive cell vector; $\vec{k}_{*}^{z}$ is the vector at the Brillouin zone boundary directed along $Z$ )

$$
\eta_{n f,-}^{A(B)}=\mathrm{e}^{\mathrm{i} \vec{n} \vec{k}_{*}^{z}} \cdot \eta_{f,-}^{A(B)}
$$

Hence, in the cases of both ADP and RDP we use a primitive cell with " $A$ " and " $B$ " tetrahedra. 
Hamiltonian of a mixed $\mathrm{Rb}_{1-x}\left(\mathrm{NH}_{4}\right)_{x} \mathrm{H}_{2} \mathrm{PO}_{4}$ system can be written as

$$
\begin{aligned}
& H(\{h\})=-\sum_{n, f}\left(\vec{d}_{n f} \cdot\left[\vec{E}+\vec{G}_{n}\right]\right) S_{n f}+\sum_{n}\left[H_{A}(n)+H_{B}(n)\right]-\frac{1}{2} \sum_{n, f} \sum_{n^{\prime}, f^{\prime}} J_{n f, n^{\prime} f^{\prime}} S_{n f} S_{n^{\prime} f^{\prime}} \\
& H_{A}(n)=\frac{V_{n}}{4}\left(S_{n 1} S_{n 2}+S_{n 2} S_{n 3}+S_{n 3} S_{n 4}+S_{n 4} S_{n 1}\right)+\frac{U_{n}}{4}\left(S_{n 1} S_{n 3}+S_{n 2} S_{n 4}\right)+\frac{\Phi_{n}}{16} S_{n 1} S_{n 2} S_{n 3} S_{n 4} .
\end{aligned}
$$

Here $S_{n f}= \pm 1$ are spin operators describing the position of a proton on the $f=1,2,3,4$ hydrogen bond in the $\vec{n}$ cell at a $R$ tetrahedron; $\vec{E}$ is an external uniform electric field; $\vec{G}_{n}$ is an internal random deformational field; $J_{n f, n^{\prime} f^{\prime}}$ is the long-range interaction between protons; $H_{A}(n), H_{B}(n)$ are the configurational energies of the " $A$ ", " $B$ " tetrahedra. In this work we take into account two configurational states of a tetrahedron $(\alpha=+,-)$

$$
V_{\alpha}=-\frac{1}{8} w_{1 \alpha}, \quad U_{\alpha}=\frac{1}{8}\left(w_{1 \alpha}-2 \varepsilon_{\alpha}\right), \quad \Phi_{\alpha}=\frac{1}{8}\left(w_{1 \alpha}+2 \varepsilon_{\alpha}-4 w_{\alpha}\right), \quad \alpha=+,-
$$

In the state + , the energy states of a tetrahedron are analogous to those in a pure RDP crystal with the ground level $\varepsilon_{s+}$

$$
\varepsilon_{+}=\varepsilon_{a+}-\varepsilon_{s+}, \quad w_{+}=\varepsilon_{1+}-\varepsilon_{s+}, \quad w_{1+}=\varepsilon_{o+}-\varepsilon_{s+} .
$$

In the state - (ADP) we use the same relations for $V_{\alpha}, U_{\alpha}, \Phi_{\alpha}$ but with different values of $\varepsilon_{\alpha}$, $w_{\alpha}, w_{1 \alpha}$.

In the case of a mixed $\mathrm{Rb}_{1-x}\left(\mathrm{NH}_{4}\right)_{x} \mathrm{H}_{2} \mathrm{PO}_{4}$ crystal, ionic positions are occupied by $\mathrm{Rb}$ with probability $c_{+}=1-x$ and by $\mathrm{NH}_{4}$ with probability $c_{-}=x$. Hence, the distribution function of a strongly random energy parameter $\varepsilon_{\alpha}$ (and similarly for $w_{\alpha}, w_{1 \alpha}$ ) can be qualitatively written as

$$
p(\sigma)=(1-x) \delta\left(\sigma-\varepsilon_{+}\right)+x \delta\left(\sigma-\varepsilon_{-}\right)=c_{+} \delta\left(\sigma-\varepsilon_{+}\right)+c_{-} \delta\left(\sigma-\varepsilon_{-}\right) .
$$

A state of a dipole moment on the bond $\vec{d}_{f, \alpha \alpha_{f}}$ is determined by the states $\alpha, \alpha_{f}$ of two tetrahedra connected by this bond. In the mean field approximation over the bonds, the average on configuration moment of a tetrahedron $\left\langle\vec{P}^{B}\right\rangle_{\mathrm{c}}$ reads

$$
\left\langle\vec{P}^{B}\right\rangle_{\mathrm{c}} \approx \sum_{f=1}^{4}\left\langle\vec{d}_{f}\right\rangle_{\mathrm{c}} \bar{\eta}_{f}, \quad\left\langle\vec{d}_{f}\right\rangle_{\mathrm{c}}=\sum_{\alpha} \sum_{\beta} c_{\alpha} c_{\beta} \vec{d}_{f, \alpha \beta}, \quad \bar{\eta}_{f}=\left\langle\left\langle S_{f}\right\rangle_{\mathrm{c}} .\right.
$$

In the present work we consider only two realizations of the sets of the averaged over configurations values of $\bar{\eta}_{f}=\bar{\eta} ; \quad-\bar{\eta}_{1,-}^{B}=\bar{\eta}_{2,-}^{B}=\bar{\eta}_{3,-}^{B}=-\bar{\eta}_{4,-}^{B}=\bar{\eta}$, which correspond to ferroelectric and antiferroelectric ordering. This permits to use the primitive cell of RDP with 2 tetrahedra and 4 hydrogen bonds. Then the mean free energy per primitive cell $\langle\mathbf{F}\rangle$ can be written as follows:

$$
\begin{aligned}
-\beta\langle\mathbf{F}\rangle= & -\sum_{f=1, \in A}^{4}\left\langle F_{f}^{(0)}\right\rangle_{\mathrm{c}}+\left\langle F_{A}^{[0]}\right\rangle_{\mathrm{c}}+\left\langle F_{B}^{[0]}\right\rangle_{\mathrm{c}}-\sum_{f=1, \in A}^{4} \varphi_{L, f}\left\langle F_{f}^{(1)}\right\rangle_{\mathrm{c}} \\
& +\frac{1}{2} \sum_{f, f^{\prime}=1, \in A}^{4}\left\langle J_{f, f^{\prime}}\left(\vec{k}_{*}\right)\right\rangle_{\mathrm{c}}\left\langle F_{f}^{(1)}\right\rangle_{\mathrm{c}}\left\langle F_{f^{\prime}}^{(1)}\right\rangle_{\mathrm{c}},
\end{aligned}
$$

where $\vec{k}_{*}=\overrightarrow{0}_{*}$ for ferroelectric ordering $\left\langle F_{f}^{(1)}\right\rangle_{\mathrm{c}}=\left\langle F^{(1)}\right\rangle_{\mathrm{c}}, \vec{k}_{*}=\vec{k}_{*}^{z}$ for antiferroelectric ordering $-\left\langle F_{1}^{(1)}\right\rangle_{\mathrm{c}}=\left\langle F_{2}^{(1)}\right\rangle_{\mathrm{c}}=\left\langle F_{3}^{(1)}\right\rangle_{\mathrm{c}}=-\left\langle F_{4}^{(1)}\right\rangle_{\mathrm{c}}^{\mathrm{c}}=\left\langle F^{(1)}\right\rangle_{\mathrm{c}}$. We use the following notations for averages over different random fields of the single-particle $F_{f}^{(0)}$ and cluster $F_{1234}^{[0000]}$ generating functions

$$
\begin{aligned}
\left\langle F_{f}^{(0)}\right\rangle_{\mathrm{c}} & =\left\langle F^{(0)}\left(\xi_{f}\right)\right\rangle_{\mathrm{c}}=\left\langle F^{(0)}\left(\kappa_{f}+\sigma+g_{x}+g_{y}+g_{z}\right)\right\rangle_{\sigma, \vec{g}} \\
& =\int \ldots \int \mathrm{d} \sigma R(\sigma, 2 q) \rho_{t}\left(g_{x}\right) \rho_{t}\left(g_{y}\right) \rho_{z}\left(g_{z}\right) \mathrm{d} g_{x} \mathrm{~d} g_{y} \mathrm{~d} g_{z} \cdot F^{(0)}\left(\kappa_{f}+\sigma+g_{x}+g_{y}+g_{z}\right),
\end{aligned}
$$




$$
\begin{aligned}
& \left\langle F_{A}^{[0]}\right\rangle_{\mathrm{c}}=\left\langle F_{1234}^{[0000]}\right\rangle_{\mathrm{c}}=\left\langle F^{[0000]}\left(\xi_{1}\left|\xi_{2}\right| \xi_{3}\left|\xi_{4}\right| \mid R\right)\right\rangle_{\mathrm{c}}=\int \ldots \int \prod_{f=1}^{4} \mathrm{~d} \sigma_{f} R\left(\sigma_{f}, q\right) \rho\left(g_{x}\right) \rho\left(g_{y}\right) \rho\left(g_{z}\right) \mathrm{d} g_{x} \mathrm{~d} g_{y} \mathrm{~d} g_{z} \\
& \times\left\langle F^{[0000]}\left(\kappa_{\mathrm{cl}, 1}+\sigma_{1}+g_{z}-g_{x}\left|\kappa_{\mathrm{cl}, 2}+\sigma_{2}+g_{z}+g_{y}\right| \kappa_{\mathrm{cl}, 3}+\sigma_{3}+g_{z}+g_{x}\left|\kappa_{\mathrm{cl}, 4}+\sigma_{2}+g_{z}-g_{y}\right| \mid R\right)\right\rangle_{\{\sigma\}, \vec{g}, R} .
\end{aligned}
$$

Here, we introduce notations for the average values of cluster $\varphi_{f}$ and long-range $\varphi_{L, f}$ fields, and

$$
\kappa_{f}=h_{f}+\bar{\varphi}_{L, f}+2 \bar{\varphi}_{f}, \quad \kappa_{\mathrm{cl}, f}=h_{f}+\bar{\varphi}_{L, f}+\bar{\varphi}_{f}, \quad h_{f}=\left(\left\langle\vec{d}_{f}\right\rangle_{c} \cdot \vec{E}\right) .
$$

Averaging is performed over random cluster fields with dispersion $q$ and over random deformational fields with dispersion $\left\langle G^{2}\right\rangle_{c}$

$$
R(\sigma, q)=\frac{1}{\sqrt{2 \pi q}} \exp \left\{-\frac{1}{2} \frac{\sigma^{2}}{q}\right\}, \quad \rho(\sigma)=\frac{1}{\sqrt{2 \pi\left\langle G^{2}\right\rangle_{c}}} \exp \left\{-\frac{1}{2} \frac{\sigma^{2}}{\left\langle G^{2}\right\rangle_{c}}\right\}, \quad\left\langle G^{2}\right\rangle_{c}=4 x(1-x) Q_{G} .
$$

The expressions for the single-particle function $F_{f}^{(0)}$ and its derivatives $F_{f}^{(n)}$ are as follows:

$$
\begin{aligned}
& F_{f}^{(0)}=\ln \left[2 \operatorname{ch}\left(\beta \xi_{f}\right)\right], \quad F_{f}^{(n)}=\partial^{n} / \partial\left(\beta \xi_{f}\right)^{n} F_{f}^{(0)}, \quad F_{f}^{(1)}=\operatorname{th}\left(\beta \xi_{f}\right) \\
& F_{f}^{(2)}=1-\left(F_{f}^{(1)}\right)^{2}, \quad F_{f}^{(3)}=-2 F_{f}^{(1)} F_{f}^{(2)}, \quad F_{f}^{(4)}=-2 F_{f}^{(2)}\left[1-3\left(F_{f}^{(1)}\right)^{2}\right] .
\end{aligned}
$$

The cluster function $F_{1234}^{[0000]}$ and its derivatives $F_{1234}^{\left[n_{1} n_{2} n_{3} n_{4}\right]} \mathrm{read}$

$$
\begin{aligned}
& F^{[0000]}\left(\xi_{1}\left|\xi_{2}\right| \xi_{3}\left|\xi_{4}\right| \mid R\right)=\ln \left[0.5 \cdot L\left(\xi_{1}, \xi_{2}, \xi_{3}, \xi_{4}|| R_{\alpha}\right)\right] \\
& F_{1234}^{\left[n_{1} n_{2} n_{3} n_{4}\right]}=\frac{\partial^{n_{1}}}{\partial\left(\beta \xi_{1}\right)^{n_{1}}} \cdots \frac{\partial^{n_{4}}}{\partial\left(\beta \xi_{1}\right)^{n_{4}}} F_{1234}^{[0000]}, \\
& F_{11}^{[1,1]}=F^{[2000]}\left(\xi_{1}\left|\xi_{2}\right| \xi_{3}\left|\xi_{4}\right| \mid R\right)=1-M_{1}^{[1]} M_{1}^{[1]}, \\
& F_{12}^{[1,1]}=F^{[1100]}\left(\xi_{1}\left|\xi_{2}\right| \xi_{3}\left|\xi_{4}\right| \mid R\right)=M_{12}^{[1,1]}-M_{1}^{[1]} M_{2}^{[1]}, \\
& F_{13}^{[1,1]}=F^{[1010]}\left(\xi_{1}\left|\xi_{2}\right| \xi_{3}\left|\xi_{4}\right| \mid R\right)=M_{13}^{[1,1]}-M_{1}^{[1]} M_{3}^{[1]}, \\
& F_{14}^{[1,1]}=F^{[1001]}\left(\xi_{1}\left|\xi_{2}\right| \xi_{3}\left|\xi_{4}\right| \mid R\right)=M_{14}^{[1,1]}-M_{1}^{[1]} M_{4}^{[1]}, \\
& F_{f f^{\prime}}^{[21]}=-2 F_{f}^{[1]} F_{f f^{\prime}}^{[11]}, \quad F_{f f^{\prime}}^{[21]}=-2 F_{f f^{\prime}}^{[11]} F_{f^{\prime}}^{[1]}, \\
& F_{f f^{\prime}}^{[22]}=-2 F_{f f^{\prime}}^{[1,1]}\left[M_{f f^{\prime}}^{[1,1]}-M_{f}^{[1]} M_{f^{\prime}}^{[1]]},\right. \\
& M_{1}^{[1]}=L_{1234}^{[1000]} / L_{1234}^{[0000]}, \ldots M_{4}^{[1]}=L_{1234}^{[0001]} / L_{1234}^{[0000]} \\
& M_{11}^{[1,1]}=L_{1234}^{[2000]} / L_{1234}^{[0000]} \equiv 1, \ldots, M_{14}^{[1,1]}=L_{1234}^{[1001]} / L_{1234}^{[0000]}, \\
& \\
& 0.5 L\left(\xi_{1}, \xi_{2}, \xi_{3}, \xi_{4}|| R_{\alpha}\right)=2 a_{\alpha} \cdot \operatorname{ch}\left(\beta \xi_{1}-\beta \xi_{3}\right) \operatorname{ch}\left(\beta \xi_{2}-\beta \xi_{4}\right) \\
&+\operatorname{ch}\left(\beta \xi_{1}+\beta \xi_{2}+\beta \xi_{3}+\beta \xi_{4}\right)+d_{\alpha} \cdot \operatorname{ch}\left(\beta \xi_{1}-\beta \xi_{2}+\beta \xi_{3}-\beta \xi_{4}\right) \\
&+ 2 b_{\alpha}\left[\operatorname{ch}\left(\beta \xi_{1}+\beta \xi_{3}\right) \operatorname{ch}\left(\beta \xi_{2}-\beta \xi_{4}\right)+\operatorname{ch}\left(\beta \xi_{1}-\beta \xi_{3}\right) \operatorname{ch}\left(\beta \xi_{2}+\beta \xi_{4}\right)\right] \\
&= \exp \left(-\beta \varepsilon_{\alpha}\right), \quad b_{\alpha}=\exp \left(-\beta w_{\alpha}\right), \quad d_{\alpha}=\exp \left(-\beta w_{1 \alpha}\right) . \\
&\left.a_{\alpha}\right)
\end{aligned}
$$


Here the partition function $0.5 L\left(\{\xi\} \| R_{\alpha}\right)$ is calculated with the cluster Hamiltonian

$$
\begin{aligned}
H_{A}\left(\{\xi\} ; S_{1}, S_{2}, S_{3}, S_{4} \| R\right)= & H_{A}\left(\{0\} ; S_{1}, S_{2}, S_{3}, S_{4} \| R\right)-\sum_{f=1}^{4} \xi_{f} S_{f}, \\
H\left(\{0\} ; S_{1}, S_{2}, S_{3}, S_{4} \| R\right)= & \frac{V_{\alpha}}{4} \cdot\left(S_{1} S_{2}+S_{2} S_{3}+S_{3} S_{4}+S_{4} S_{1}\right) \\
& +\frac{U_{\alpha}}{4} \cdot\left(S_{1} S_{3}+S_{2} S_{4}\right)+\frac{\Phi_{\alpha}}{16} \cdot S_{1} S_{2} S_{3} S_{4} .
\end{aligned}
$$

We shall use the same model dependence of the average eigenvalues of the long-range interaction matrix as for the dipole moment of a hydrogen bond:

$$
\left\langle\nu_{\mu}\left(\vec{k}_{*}\right)\right\rangle_{\mathrm{c}}=\bar{\nu}_{\mu}\left(\vec{k}_{*}\right)=\sum_{\alpha} \sum_{\beta} c_{\alpha} c_{\beta} \bar{\nu}_{\mu, \alpha \beta}\left(\vec{k}_{*}\right) \approx c_{+}^{2} \bar{\nu}_{\mu,++}\left(\vec{k}_{*}\right)+c_{-}^{2} \bar{\nu}_{\mu,--}\left(\vec{k}_{*}\right)+2 c_{+} c_{-} \bar{\nu}_{\mu, 00}\left(\vec{k}_{*}\right) .
$$

For these values of $\vec{k}_{*}$ the long-range interaction matrix $\bar{J}_{f, f^{\prime}}=\left\langle J_{f, f^{\prime}}\left(\vec{k}_{*}\right)\right\rangle_{c}$ and the unitary transformation matrix $U=\left\{u_{\mu f}\right\}$ read

$$
\begin{aligned}
& U=U^{+}=\frac{1}{2}\left(\begin{array}{llll}
1 & 1 & 1 & 1 \\
1 & 1 & -1 & -1 \\
1 & -1 & 1 & -1 \\
1 & -1 & -1 & 1
\end{array}\right) ; \quad\langle J\rangle=\left(\begin{array}{llll}
\bar{J}_{11} & \bar{J}_{12} & \bar{J}_{13} & \bar{J}_{12} \\
\bar{J}_{12} & \bar{J}_{11} & \bar{J}_{12} & \bar{J}_{13} \\
\bar{J}_{13} & \bar{J}_{12} & \bar{J}_{11} & \bar{J}_{12} \\
\bar{J}_{12} & \bar{J}_{13} & \bar{J}_{12} & \bar{J}_{11}
\end{array}\right) \\
& \hat{\bar{\nu}}=\hat{U} \hat{\bar{J}} \hat{U}=\left(\begin{array}{llll}
\bar{\nu}_{1} & 0 & 0 & 0 \\
0 & \bar{\nu}_{2} & 0 & 0 \\
0 & 0 & \bar{\nu}_{3} & 0 \\
0 & 0 & 0 & \bar{\nu}_{4}
\end{array}\right) \\
& \bar{\nu}_{1}=\bar{J}_{11}+2 \bar{J}_{12}+\bar{J}_{13} ; \quad \bar{\nu}_{2}=\bar{\nu}_{4}=\bar{J}_{11}-\bar{J}_{13} ; \quad \bar{\nu}_{3}=\bar{J}_{11}-2 \bar{J}_{12}+\bar{J}_{13} .
\end{aligned}
$$

From the condition of the free energy extremum we find an expression for the average $\bar{\eta}_{f}=$ $\left\langle\left\langle S_{n f}\right\rangle\right\rangle_{\mathrm{c}}$, reduced Edwards-Anderson parameter $Q_{\mathrm{EA}, f}$ and an equation for unknown quantities $\bar{\varphi}_{L, f}, \bar{\varphi}_{f}, q_{f}$

$$
\begin{aligned}
& \bar{\eta}_{f}=\left\langle F_{f}^{(1)}\right\rangle, \quad Q_{\mathrm{EA}, f}=q_{\mathrm{EA}, f}-\bar{\eta}_{f}^{2}, \quad q_{\mathrm{EA}, f}=1-\left\langle F_{f}^{(2)}\right\rangle \\
& \left\langle F_{f}^{(1)}\right\rangle=\left\langle F_{f}^{[1000]}\right\rangle, \quad\left\langle F_{f}^{(2)}\right\rangle=\left\langle F_{f}^{[2000]}\right\rangle, \quad \bar{\varphi}_{L, f}=\sum_{f_{1}}^{4} \bar{J}_{f f_{1}}\left(\vec{k}_{*}\right) \bar{\eta}_{f_{1}} .
\end{aligned}
$$

In the absence of external field and for the ferroelectric ordering we obtain the following expressions for the free energy, for the average $\bar{\eta}=\bar{\eta}_{f}$, reduced Edwards-Anderson parameter $Q_{\mathrm{EA}}=Q_{\mathrm{EA}, f}$ and for equations for $\bar{\varphi}_{L}, \bar{\varphi}, q$

$$
\begin{aligned}
\bar{\eta} & =\bar{\eta}_{f}, \quad \bar{\varphi}=\bar{\varphi}_{f}, \quad \bar{\varphi}_{L}=\bar{\varphi}_{L, f}, \quad q=q_{f}, \\
-\beta\left\langle\mathbf{F}_{F}\right\rangle & =-4\left\langle F^{(0)}\right\rangle+2\left\langle F_{A}\right\rangle-4 \bar{\varphi}_{L}\left\langle F^{(1)}\right\rangle+2 \bar{\nu}_{1}\left(\overrightarrow{0}_{*}\right)\left\langle F^{(1)}\right\rangle^{2}, \\
\bar{\eta} & =\left\langle F^{(1)}\right\rangle, \quad Q_{\mathrm{EA}}=q_{\mathrm{EA}}-\bar{\eta}^{2}, \quad q_{\mathrm{EA}, f}=1-\left\langle F^{(2)}\right\rangle, \\
\left\langle F^{(1)}\right\rangle & =\left\langle F^{[1000]}\right\rangle, \quad\left\langle F^{(2)}\right\rangle=\left\langle F^{[2000]}\right\rangle, \quad \bar{\varphi}_{L}=\bar{\nu}_{1}\left(\overrightarrow{0}_{*}\right) \bar{\eta} .
\end{aligned}
$$

In the case of an antiferroelectric ordering in the absence of external field the free energy, for the average $\bar{\eta}=-\bar{\eta}_{1}=\bar{\eta}_{2}$, reduced Edwards-Anderson parameter $Q_{\mathrm{EA}}=Q_{\mathrm{EA}, f}$ and equations for 
$\bar{\varphi}_{L}, \bar{\varphi}, q \mathrm{read}$

$$
\begin{aligned}
\bar{\eta} & =-\bar{\eta}_{1(4)}=\eta_{2(3)}, \bar{\varphi}=-\bar{\varphi}_{1(4)}=\bar{\varphi}_{2(3)}, \bar{\varphi}_{L}=-\bar{\varphi}_{L, 1(4)}=\bar{\varphi}_{L, 2(3)}, q=q_{f}, \\
-\beta\left\langle\mathbf{F}_{A F}\right\rangle & =-4\left\langle F^{(0)}\right\rangle+2\left\langle F_{A}\right\rangle-4 \bar{\varphi}_{L}\left\langle F^{(1)}\right\rangle+2 \nu_{2}\left(\vec{k}_{*}^{z}\right)\left\langle F^{(1)}\right\rangle^{2}, \\
\bar{\eta} & =\left\langle F^{(1)}\right\rangle=-\left\langle F_{1}^{(1)}\right\rangle, \quad Q_{\mathrm{EA}}=q_{\mathrm{EA}}-\bar{\eta}^{2}, \quad q_{\mathrm{EA}, f}=1-\left\langle F^{(2)}\right\rangle, \\
\left\langle F^{(1)}\right\rangle & =\left\langle F^{[0100]}\right\rangle, \quad\left\langle F^{(2)}\right\rangle=\left\langle F^{[0200]}\right\rangle, \quad \bar{\varphi}_{L}=\bar{\nu}_{2}\left(\vec{k}_{*}^{z}\right) \bar{\eta} .
\end{aligned}
$$

As numerical calculations for the free energy show, the antiferroelectric state is realized in the region close to the $x=1-c \rightarrow 1$ limit, the ferroelectric state is realized in the region $1-x=c \rightarrow 1$, and a proton glass state $\left(\bar{\varphi}=\bar{\varphi}_{L}=0, q>0\right)$ takes place at intermediate compositions.

The static susceptibility of the system reads ( $v_{e}$ is the cell volume)

$$
\begin{aligned}
& \chi_{a b}=-\frac{1}{T v_{e}} \sum_{f, f^{\prime}} \bar{d}_{f}^{a} \bar{d}_{f^{\prime}}^{b} \cdot \bar{\eta}_{f f^{\prime}}^{\prime}=-\frac{1}{T v_{e}} \sum_{\mu} \tilde{\bar{d}}_{\mu}^{a} \tilde{\bar{d}}_{\mu}^{b} \cdot \tilde{\bar{\eta}}_{\mu}^{\prime}, \\
& \bar{\eta}_{f f^{\prime}}^{\prime}=\frac{\partial \bar{\eta}_{f}}{\partial h_{f^{\prime}}} ; \quad \tilde{\bar{d}}_{\mu}^{a}=\sum_{f} u_{\mu f} \bar{d}_{f}^{a} ; \quad \tilde{\bar{\eta}}_{\mu}^{\prime}=\sum_{f, f^{\prime}} u_{\mu f} u_{\mu f^{\prime}} \bar{\eta}_{f f^{\prime}}^{\prime} .
\end{aligned}
$$

Here we used the fact that after the unitary transformation with $\hat{U}$, the matrix of $\tilde{\bar{\eta}}_{\mu \mu^{\prime}}^{\prime}=\delta_{\mu \mu^{\prime}} \tilde{\bar{\eta}}_{\mu}^{\prime}$ correlators is diagonal for ferroelectric and antiferroelectric orderings, and

$$
\begin{array}{ll}
\tilde{\bar{\eta}}_{1}^{\prime}=\bar{\eta}_{11}^{\prime}+\bar{\eta}_{12}^{\prime}+\bar{\eta}_{13}^{\prime}+\bar{\eta}_{14}^{\prime}, & \tilde{\bar{\eta}}_{2}^{\prime}=\bar{\eta}_{11}^{\prime}+\bar{\eta}_{12}^{\prime}-\bar{\eta}_{13}^{\prime}-\bar{\eta}_{14}^{\prime}, \\
\tilde{\bar{\eta}}_{3}^{\prime}=\bar{\eta}_{11}^{\prime}-\bar{\eta}_{12}^{\prime}+\bar{\eta}_{13}^{\prime}-\bar{\eta}_{14}^{\prime}, & \tilde{\bar{\eta}}_{4}^{\prime}=\bar{\eta}_{11}^{\prime}-\bar{\eta}_{12}^{\prime}-\bar{\eta}_{13}^{\prime}+\bar{\eta}_{14}^{\prime} .
\end{array}
$$

We shall be interested in the longitudinal $\chi_{33}$ and transverse $\chi_{a a}$ susceptibilities of the system

$$
\chi_{33}=-4 \frac{1}{T v_{e}}\left(\bar{d}^{z}\right)^{2} \cdot \tilde{\bar{\eta}}_{1}^{\prime}, \quad \chi_{a a}=-\frac{1}{T v_{e}}\left(\bar{d}^{a}\right)^{2} \cdot\left(\tilde{\bar{\eta}}_{2}^{\prime}+\tilde{\bar{\eta}}_{4}^{\prime}\right), \quad a=1,2 .
$$

\section{Relaxational dynamics of the $\mathrm{Rb}_{1-x}\left(\mathrm{NH}_{4}\right)_{x} \mathrm{H}_{2} \mathrm{PO}_{4}$ type mixtures}

Dynamics of the mixed system $\mathrm{Rb}_{1-x}\left(\mathrm{NH}_{4}\right)_{x} \mathrm{H}_{2} \mathrm{PO}_{4}$ will be described on the basis of Glauber equations for the $n$-th order correlation functions:

$$
\begin{aligned}
& \left(\sum_{j=1}^{n} \nu_{j}+\partial / \partial t\right) \eta_{12 \ldots n}(t)=\sum_{j}^{n} \nu_{j}\left\langle S_{1} \ldots S_{n} F^{(1)}\left(h_{j}(t)+\bar{\varphi}_{L, j}(t)+\hat{\varphi}_{j}^{-}+\hat{\varphi}_{j}^{+}\right)\right\rangle_{\rho(t)}, \\
& \eta_{12 \ldots n}(t)=\left\langle S_{1} \ldots S_{n}\right\rangle_{\rho(t)}, \quad \nu_{j}=1 / \tau_{0, j}, \quad \bar{\varphi}_{L, j}(t)=\sum_{j} \bar{J}_{j j^{\prime}}\left(\vec{k}_{*}\right) \bar{\eta}_{j}^{\prime}(t) .
\end{aligned}
$$

Here we introduce operator fields $\varphi_{j}^{+}, \varphi_{j}^{-}$exerted on the bond $j$ by the two tetrahedra connected by this bond

$$
\begin{aligned}
& \hat{\varphi}_{j}^{+}=-V_{j} / 4\left(S_{j_{2}}+S_{j_{4}}\right)-U_{j} / 4 S_{j_{3}}-\Phi_{j} / 16 S_{j_{2}} S_{j_{3}} S_{j_{4}}, \\
& \hat{\varphi}_{j}^{-}=-V_{j} / 4\left(S_{j_{2}^{\prime}}+S_{j_{4}^{\prime}}\right)-U_{j} / 4 S_{j_{3}^{\prime}}-\Phi_{j} / 16 S_{j_{2}^{\prime}} S_{j_{3}^{\prime}} S_{j_{4}^{\prime}} .
\end{aligned}
$$

The seed relaxation time $\tau_{0, j}$ can be expressed via correlation functions of an ionic subsystem. Within the cluster approximation given below, the bonds $i, j$ belong to the same tetrahedron (as an example we use the " $B$ " tetrahedron with indices $f, f^{\prime}=1 \div 4$ ). The averaging over configurations will also be performed for each tetrahedron with its hydrogen bonds independently. Therefore, in our equations there is no primitive cell index. 
We shall explain the approximation using the example of an equation for a single-particle correlator. In the mean field approximation we make a replacement $\hat{\varphi}_{j}^{ \pm} \rightarrow \varphi_{j}(t)$ and obtain an equation

$$
\left[\nu_{1}+\partial / \partial t\right] \cdot \eta_{1}(t)=\nu_{1} \cdot F^{(1)}\left(\kappa_{1}(t)\right), \quad \kappa_{1}(t)=h_{1}(t)+2 \varphi_{1}(t)+\bar{\varphi}_{L, 1}(t)
$$

Within the cluster approximation such a replacement is performed for a field $\hat{\varphi}_{f}^{-} \rightarrow \varphi_{f}$ only

$$
\begin{aligned}
& {\left[\nu_{1}+\partial / \partial t\right] \cdot \eta_{1}(t)=\nu_{1} \cdot F^{(1)}\left(\kappa_{\mathrm{cl}, 1}(t)+\varphi_{1}^{+}\right), \quad \kappa_{\mathrm{cl}, 1}(t)=h_{1}(t)+\varphi_{1}(t)+\bar{\varphi}_{L, 1}(t),} \\
& \begin{aligned}
\nu_{1} \cdot F^{(1)}\left(\kappa_{\mathrm{cl}, 1}(t)+\varphi_{1}^{+}\right)= & L_{1}+P_{1} \cdot \eta_{3}(t)+Q_{1}\left(\eta_{2}(t)+\eta_{4}(t)\right)+N_{1} \eta_{24}(t) \\
& +M_{1}\left(\eta_{23}(t)+\eta_{34}(t)\right)+R_{1} \eta_{234}(t) .
\end{aligned}
\end{aligned}
$$

The expansion coefficients are found from the relations

$$
\begin{aligned}
& L_{1}(t)=\frac{\nu_{1}}{8} \sum_{2,3,4} F_{1}^{(1)}, \quad P_{1}(t)=\frac{\nu_{1}}{8} \sum_{2,3,4} S_{3} F_{1}^{(1)} \\
& Q_{1}(t)=\frac{\nu_{1}}{8} \sum_{2,3,4} S_{2} F_{1}^{(1)} \equiv \frac{\nu_{1}}{8} \sum_{2,3,4} S_{2} F_{1}^{(1)}, \quad N_{1}(t)=\frac{\nu_{1}}{8} \sum_{2,3,4} S_{2} S_{4} F_{1}^{(1)} \\
& M_{1}(t)=\frac{\nu_{1}}{8} \sum_{2,3,4} S_{2} S_{3} F_{1}^{(1)}=\frac{\nu_{1}}{8} \sum_{2,3,4} S_{4} S_{3} F_{1}^{(1)}, \quad R_{1}(t)=\frac{\nu_{1}}{8} \sum_{2,3,4} S_{2} S_{3} S_{4} F_{1}^{(1)} .
\end{aligned}
$$

In equations (30), (31) the $t$-dependent distribution functions $\eta_{f}(t), \eta_{f g}(t), \eta_{f g k}(t)$ and random dynamic cluster field $\varphi_{f}(t)$ are unknown. In the cluster approximation we need to write down a system of 14 closed equations for 14 unknown correlation functions. In the matrix form

$$
\hat{A}\left(\left\{\nu_{f}\right\} ;\left\{L_{f}, P_{f}, Q_{f}, N_{f}, M_{f}, R_{f}\right\} ; \partial / \partial t\right) \cdot \vec{\eta}(t)=\vec{C}\left(\left\{L_{f}, P_{f}, Q_{f}, N_{f}, M_{f}, R_{f}\right\}\right) .
$$

Here we introduce notations for the column vectors

$$
\begin{aligned}
& \vec{\eta}(t)=\left(\eta_{1} ; \eta_{2} ; \eta_{3} ; \eta_{4}\left|\eta_{23} ; \eta_{34} ; \eta_{41} ; \eta_{12} ; \eta_{24} ; \eta_{13}\right| \eta_{234} ; \eta_{341} ; \eta_{412} ; \eta_{123}\right), \\
& -\vec{C}\left(\left\{L_{f}, P_{f}, Q_{f}, N_{f}, M_{f}, R_{f}\right\}\right)=\left(L_{1} ; L_{2} ; L_{3} ; L_{4} \mid Q_{2}+Q_{3} ; Q_{3}+Q_{4} ; Q_{1}+Q_{4} ; Q_{1}+Q_{2},\right. \\
& \left.\quad P_{2}+P_{4} ; P_{1}+P_{3} \mid M_{2}+M_{4}+N_{3} ; M_{1}+M_{3}+N_{4} ; M_{2}+M_{4}+N_{1} ; M_{1}+M_{3}+N_{2}\right) .
\end{aligned}
$$

The matrix contains the $\partial / \partial t$ operators only in its diagonal elements. It is too cumbersome to be presented here.

In the present work we are interested in the linear response of the system to a frequencydependent field

$$
\begin{aligned}
& h_{f}(t)=h_{f}+\delta h_{f}(t), \quad \kappa_{f}(t)=\kappa_{f}+\delta \kappa_{f}(t), \quad \kappa_{\mathrm{cl}, f}(t)=\kappa_{\mathrm{cl}, f}+\delta \kappa_{\mathrm{cl}, f}(t), \\
& \eta_{f f^{\prime}}^{\prime}(t)=\frac{\delta \eta_{f}(t)}{\delta h_{f^{\prime}}(t)}, \quad \kappa_{f f^{\prime}}^{\prime}(t)=\frac{\delta \kappa_{f}(t)}{\delta h_{f^{\prime}}(t)}, \quad \varphi_{f f^{\prime}}^{\prime}(t)=\frac{\delta \varphi_{f}(t)}{\delta h_{f^{\prime}}(t)} .
\end{aligned}
$$

Expanding (30) in powers of $\delta \kappa_{f}(t)$ and differentiating the dynamic part with respect to $\delta h_{f^{\prime}}(t)$, we obtain expressions for the static $\eta_{f}$ and dynamic $\eta_{f f^{\prime}}^{\prime}(\omega)$ (after the Fourier transformation $t \rightarrow \omega)$ parts

$$
\begin{aligned}
& \eta_{f}=F_{f}^{(1)}\left(\kappa_{f}\right), \quad \kappa_{f}=h_{f}+2 \varphi_{f}+\sum_{f_{1}} \bar{J}_{f f_{1}}\left(\vec{k}_{*}\right) \bar{\eta}_{f_{1}}, \\
& \eta_{f f^{\prime}}^{\prime}(\omega)=F_{f}^{(2)}(\omega) \cdot \kappa_{f f^{\prime}}^{\prime}(\omega), \quad F_{f}^{(2)}(\omega)=\nu_{f} F_{f}^{(2)}\left(\kappa_{f}\right) /\left(\nu_{f}+\mathrm{i} \omega\right), \\
& \bar{\kappa}_{f f^{\prime}}^{\prime}(\omega)=\delta_{f f^{\prime}}+2 \varphi_{f f^{\prime}}^{\prime}(\omega)+\sum_{f_{1}=1}^{4} \bar{J}_{f f_{1}}(\overrightarrow{0}) \cdot \bar{\eta}_{f_{1} f^{\prime}}^{\prime}(\omega) .
\end{aligned}
$$


Static solutions of the cluster equation (33), apparently, have the form

$$
\begin{aligned}
\vec{\eta}_{0} & =\left(\hat{A}_{0}\left(\left\{\nu_{f}\right\} ;\{\} ; 0\right)\right)^{-1} \cdot \vec{C}_{0}(\{\}), \\
\hat{A}_{0}\left(\left\{\nu_{f}\right\} ;\{\} ; 0\right) & =\hat{A}\left(\left\{\nu_{f}\right\} ;\left\{L_{f}, P_{f}, Q_{f}, N_{f}, M_{f}, R_{f}\right\}_{h_{f}(t)=h_{f}} ; 0\right), \\
\vec{C}_{0}(\{\}) & =\vec{C}\left(\left\{L_{f}, P_{f}, Q_{f}, N_{f}, M_{f}, R_{f}\right\}_{h_{f}(t)=h_{f}}\right) .
\end{aligned}
$$

Linear dynamic response can be expressed via the static solutions $\vec{\eta}_{0}$ as

$$
\left.\left.\delta \vec{\eta}(\omega)=\left(\hat{A}_{0}\left(\left\{\nu_{f}\right\} ;\{\} ; i \omega\right)\right)\right)^{-1}\left[\delta \vec{C}(\{\})-\delta \hat{A}\left(\left\{\nu_{f}\right\} ;\{\} ; \mathrm{i} \omega\right)\right) \cdot \vec{\eta}_{0}\right] .
$$

Expanding the linear responses $\left.\delta \vec{C}(\{\}), \delta \hat{A}\left(\left\{\nu_{f}\right\} ;\{\} ; \mathrm{i} \omega\right)\right)$ in powers of $\delta \kappa_{\mathrm{cl}, f}(\mathrm{i} \omega)$ and differentiating with respect to $\delta h_{f}^{\prime}(\omega)$, we obtain an expression for $\eta_{f f^{\prime}}^{\prime}(\omega)$

$$
\begin{aligned}
\eta_{f f^{\prime}}^{\prime}(\omega) & =\sum_{f_{1}=1}^{4} \Omega_{f f_{1}}(\omega) \kappa_{\mathrm{cl}, f_{1} f^{\prime}}^{\prime}(\omega), \quad \kappa_{\mathrm{cl}, f f^{\prime}}^{\prime}(\omega)=\delta_{f f^{\prime}}+\varphi_{f f^{\prime}}^{\prime}(\omega)+\sum_{f_{1}=1}^{4} \bar{J}_{f f_{1}}(\overrightarrow{0}) \cdot \bar{\eta}_{f_{1} f^{\prime}}^{\prime}(\omega) \\
\Omega_{f f^{\prime}}(\omega) & =\sum_{i=1}^{14}\left(A_{0}^{-1}\right)_{f, i} \cdot \frac{\partial\left(C_{0}\right)_{i}}{\partial \kappa_{\mathrm{cl}, f^{\prime}}}-\sum_{i, j=1}^{14}\left(A_{0}^{-1}\right)_{f, i} \cdot \frac{\partial\left(A_{0}\right)_{i, j}}{\partial \kappa_{\mathrm{cl}, f^{\prime}}} \cdot \eta_{0, j}
\end{aligned}
$$

After averaging over configurations and taking into account the Gaussian fluctuations, we obtain from (36) and (39)

$$
\begin{aligned}
\bar{\eta}_{f f^{\prime}}^{\prime}(\omega)= & \left\langle\eta_{f f^{\prime}}^{\prime}(\omega)\right\rangle_{\mathrm{c}}=\left\langle F_{f}^{(2)}(\omega)\right\rangle_{\mathrm{c}}\left[\delta_{f f^{\prime}}+2 \bar{\varphi}_{f f^{\prime}}^{\prime}(\omega)+\sum_{f_{1}=1}^{4} \bar{J}_{f f_{1}}(\overrightarrow{0}) \cdot \bar{\eta}_{f_{1} f^{\prime}}^{\prime}(\omega)\right] \\
& +\left\langle F_{f}^{(3)}(\omega)\right\rangle_{\mathrm{c}} q_{f f^{\prime}}^{\prime}(\omega), \\
\bar{\eta}_{f f^{\prime}}^{\prime}(\omega)= & \sum_{f_{1}=1}^{4}\left\langle\Omega_{f f_{1}}(\omega)\right\rangle_{\mathrm{c}}\left[\delta_{f_{1} f^{\prime}}+\bar{\varphi}_{f_{1} f^{\prime}}^{\prime}(\omega)+\sum_{f_{2}=1}^{4} \bar{J}_{f_{1} f_{2}}(\overrightarrow{0}) \cdot \bar{\eta}_{f_{2} f^{\prime}}^{\prime}(\omega)\right] \\
& +\sum_{f_{1}=1}^{4}\left\langle\Omega_{f f_{1}}^{\prime}(\omega)\right\rangle_{\mathrm{c}} \frac{1}{2} q_{f_{1} f^{\prime}}^{\prime}(\omega) .
\end{aligned}
$$

Here we introduce the notations

$$
\left\langle\varphi_{f_{1}} \cdot \varphi_{f_{1} f^{\prime}}^{\prime}(\omega)\right\rangle_{\mathrm{c}}^{\mathrm{cum}}=q_{f_{1} f^{\prime}}^{\prime}(\omega) / 2 ; \quad\left\langle\Omega_{f f^{\prime}}^{\prime}(\omega)\right\rangle_{\mathrm{c}}=\left\langle\frac{\partial \Omega_{f f^{\prime}}(\omega)}{\partial \kappa_{\mathrm{cl}, f^{\prime}}}\right\rangle_{\mathrm{c}} .
$$

Equating the mean values of correlators $\bar{\eta}_{f f^{\prime}}^{\prime}(\omega)$ calculated within the single-particle and cluster approximations, we obtain the first matrix equation for unknown matrices $\hat{\varphi}^{\prime}(\omega), \hat{q}^{\prime}(\omega)$

$$
\begin{gathered}
{\left[2\left\langle\hat{F}^{(2)}(\omega)\right\rangle_{\mathrm{c}}-\langle\hat{\Omega}(\omega)\rangle_{\mathrm{c}}\right] \cdot \hat{\bar{\varphi}}^{\prime}(\omega)+\frac{1}{2}\left[2\left\langle\hat{F}^{(3)}(\omega)\right\rangle_{\mathrm{c}}-\left\langle\hat{\Omega}^{\prime}(\omega)\right\rangle_{\mathrm{c}}\right] \cdot \hat{q}^{\prime}(\omega)} \\
=\left[-\left\langle\hat{F}^{(2)}(\omega)\right\rangle_{\mathrm{c}}+\langle\hat{\Omega}(\omega)\rangle_{\mathrm{c}}\right] \cdot\left[1+\hat{\bar{J}}(\overrightarrow{0}) \hat{\bar{\eta}}^{\prime}(\omega)\right] .
\end{gathered}
$$

Equating the mean values of correlators $\left\langle Q_{f f^{\prime}}^{\prime}(\omega)\right\rangle_{\mathrm{c}}=-2\left\langle\eta_{0, f} \cdot \eta_{f f^{\prime}}^{\prime}(\omega)\right\rangle_{\mathrm{c}}$ calculated within the single-particle and cluster approximations, we obtain the second matrix equation for unknown matrices $\hat{\varphi}^{\prime}(\omega), \hat{q}^{\prime}(\omega)$

$$
\begin{gathered}
{\left[2\left\langle\hat{F}^{(3)}(\omega)\right\rangle_{\mathrm{c}}-\left\langle\hat{\Omega}_{Q}(\omega)\right\rangle_{\mathrm{c}}\right] \cdot \hat{\bar{\varphi}}^{\prime}(\omega)+\frac{1}{2}\left[2\left\langle\hat{F}^{(4)}(\omega)\right\rangle_{\mathrm{c}}-\left\langle\hat{\Omega}_{Q}^{\prime}(\omega)\right\rangle_{\mathrm{c}}\right] \cdot \hat{q}^{\prime}(\omega)} \\
=\left[-\left\langle\hat{F}^{(3)}(\omega)\right\rangle_{\mathrm{c}}+\left\langle\hat{\Omega}_{Q}(\omega)\right\rangle_{\mathrm{c}}\right] \cdot\left[1+\hat{\bar{J}}(\overrightarrow{0}) \hat{\bar{\eta}}^{\prime}(\omega)\right] .
\end{gathered}
$$


Here we introduce matrix notations

$$
\left\langle\Omega_{Q, f f^{\prime}}(\omega)\right\rangle_{\mathrm{c}}=\left\langle-2 \eta_{0, f} \cdot \Omega_{f f^{\prime}}(\omega)\right\rangle_{\mathrm{c}}, \quad\left\langle\Omega_{Q, f f^{\prime}}^{\prime}(\omega)\right\rangle_{\mathrm{c}}=\left\langle\frac{\partial \Omega_{Q, f f^{\prime}}(\omega)}{\partial \kappa_{\mathrm{cl}, f^{\prime}}}\right\rangle_{\mathrm{c}}
$$

From equations (42), (43) we derive expressions for $\hat{\varphi}^{\prime}(\omega), \hat{q}^{\prime}(\omega)$ used to find $\bar{\eta}_{f f^{\prime}}^{\prime}(\omega)(40)$. For the sake of simplicity, in what follows we shall use an effective relaxation time $\bar{\tau}_{0}$

$$
\frac{1}{\bar{\tau}_{0}}=\left\langle\nu_{f}\right\rangle_{\mathrm{c}} \approx c_{+}^{2} \frac{1}{\tau_{0,+}}+c_{-}^{2} \frac{1}{\tau_{0,-}}+2 c_{+} c_{-} \frac{1}{\tau_{0,0}} .
$$

Let us consider the symmetry of matrices, entering these equations in the case of antiferroelectric ordering (in the case of ferroelectric ordering all minus signs should be replaced by plus signs), as well as the form of the matrices after a unitary transformation (for the sake of simplicity we omit the argument of $\omega$ in the matrix elements)

$$
\begin{aligned}
& \left\langle\hat{F}^{(2 n)}(\omega)\right\rangle_{\mathrm{c}}=\left\langle F^{(2 n)}(\omega)\right\rangle_{\mathrm{c}} \cdot \hat{I} ; \quad\left\langle\widehat{\hat{F}^{(2 n)}(\omega)}\right\rangle_{\mathrm{c}}=U^{+} \cdot\left\langle\hat{F}^{(2 n)}(\omega)\right\rangle_{\mathrm{c}} \cdot U \equiv\left\langle\hat{F}^{(2 n)}(\omega)\right\rangle_{\mathrm{c}}, \\
& \left\langle\hat{F}^{(2 n+1)}(\omega)\right\rangle_{\mathrm{c}}=\left\langle F^{(2 n+1)}(\omega)\right\rangle_{\mathrm{c}}\left(\begin{array}{llll}
1 & 0 & 0 & 0 \\
0 & -1 & 0 & 0 \\
0 & 0 & -1 & 0 \\
0 & 0 & 0 & 1
\end{array}\right) \\
& \left\langle\hat{F}^{(2 n+1)}(\omega)\right\rangle_{\mathrm{c}}=\left\langle F^{(2 n+1)}(\omega)\right\rangle_{\mathrm{c}}\left(\begin{array}{cccc}
0 & 0 & 0 & 1 \\
0 & 0 & 1 & 0 \\
0 & 1 & 0 & 0 \\
1 & 0 & 0 & 0
\end{array}\right) \\
& \hat{\bar{\varphi}}^{\prime}(\omega)=\left(\begin{array}{cccc}
\bar{\varphi}_{11}^{\prime} & \bar{\varphi}_{12}^{\prime} & \bar{\varphi}_{13}^{\prime} & \bar{\varphi}_{14}^{\prime} \\
\varphi_{12}^{\prime} & \varphi_{11}^{\prime} & \varphi_{14}^{\prime} & \varphi_{13}^{\prime} \\
\varphi_{13}^{\prime} & \varphi_{14}^{\prime} & \varphi_{11}^{\prime} & \varphi_{12}^{\prime} \\
\varphi_{14}^{\prime} & \varphi_{13}^{\prime} & \varphi_{12}^{\prime} & \varphi_{11}^{\prime}
\end{array}\right) ; \quad \tilde{\hat{\varphi}^{\prime}(\omega)}=\hat{U} \hat{\varphi^{\prime}}(\omega) \hat{U}=\left(\begin{array}{llll}
\tilde{\varphi}_{1}^{\prime} & 0 & 0 & 0 \\
0 & \tilde{\varphi}_{2}^{\prime} & 0 & 0 \\
0 & 0 & \tilde{\varphi}_{3}^{\prime} & 0 \\
0 & 0 & 0 & \tilde{\varphi}_{4}^{\prime}
\end{array}\right) \\
& \tilde{\varphi}_{1}^{\prime}(\omega)=\bar{\varphi}_{11}^{\prime}+\bar{\varphi}_{12}^{\prime}+\bar{\varphi}_{13}^{\prime}+\bar{\varphi}_{14}^{\prime} ; \quad \tilde{\varphi}_{2}^{\prime}(\omega)=\bar{\varphi}_{11}^{\prime}+\bar{\varphi}_{12}^{\prime}-\bar{\varphi}_{13}^{\prime}-\bar{\varphi}_{14}^{\prime} ; \\
& \tilde{\varphi}_{3}^{\prime}(\omega)=\bar{\varphi}_{11}^{\prime}-\bar{\varphi}_{12}^{\prime}+\bar{\varphi}_{13}^{\prime}-\bar{\varphi}_{14}^{\prime} ; \quad \tilde{\varphi}_{4}^{\prime}(\omega)=\bar{\varphi}_{11}^{\prime}-\bar{\varphi}_{12}^{\prime}-\bar{\varphi}_{13}^{\prime}+\bar{\varphi}_{14}^{\prime} ; \\
& \hat{q^{\prime}}(\omega)=\left(\begin{array}{cccc}
q_{11}^{\prime} & -q_{12}^{\prime} & -q_{13}^{\prime} & q_{14}^{\prime} \\
q_{12}^{\prime} & -q_{11}^{\prime} & -q_{14}^{\prime} & q_{13}^{\prime} \\
q_{13}^{\prime} & -q_{14}^{\prime} & -q_{11}^{\prime} & q_{12}^{\prime} \\
q_{14}^{\prime} & -q_{13}^{\prime} & -q_{12}^{\prime} & q_{11}^{\prime}
\end{array}\right) ; \quad \widetilde{q^{\prime}(\omega)}=\hat{U} \hat{q^{\prime}}(\omega) \hat{U}=\left(\begin{array}{llll}
0 & 0 & 0 & \tilde{q}_{4}^{\prime} \\
0 & 0 & \tilde{q}_{3}^{\prime} & 0 \\
0 & \tilde{q}_{2}^{\prime} & 0 & 0 \\
\tilde{q}_{1}^{\prime} & 0 & 0 & 0
\end{array}\right) \\
& \tilde{q}_{1}^{\prime}(\omega)=q_{11}^{\prime}-q_{12}^{\prime}-q_{13}^{\prime}+q_{14}^{\prime}, \quad \tilde{q}_{2}^{\prime}(\omega)=q_{11}^{\prime}-q_{12}^{\prime}+q_{13}^{\prime}-q_{14}^{\prime}, \\
& \tilde{q}_{3}^{\prime}(\omega)=q_{11}^{\prime}+q_{12}^{\prime}-q_{13}^{\prime}-q_{14}^{\prime}, \quad \tilde{q}_{4}^{\prime}(\omega)=q_{11}^{\prime}+q_{12}^{\prime}+q_{13}^{\prime}+q_{14}^{\prime} \text {. }
\end{aligned}
$$

The symmetry of $\langle\hat{\Omega}(\omega)\rangle_{\mathrm{c}},\left\langle\hat{\Omega}_{Q}^{\prime}(\omega)\right\rangle_{\mathrm{c}}$ matrices in the antiferroelectric phase is the same as of $\hat{\bar{\varphi}}^{\prime}(\omega)$, and their eigenvalues $\tilde{\Omega}_{\mu}^{\mathrm{c}}(\omega), \tilde{\Omega}_{Q, \mu}^{\prime}(\omega)$ can be written as linear combinations similar to $\tilde{\varphi}_{\mu}^{\prime}(\omega)$. Symmetry of the $\left\langle\hat{\Omega}^{\prime}(\omega)\right\rangle_{\mathrm{c}},\left\langle\hat{\Omega}_{Q}(\omega)\right\rangle_{\mathrm{c}}$ matrices coincides with the symmetry of a matrix transposed to $\hat{q}^{\prime}(\omega)$; after the unitary transformation, its form is analogous to the transposed $\widetilde{\hat{q}^{\prime}(\omega)}$ matrix with the corresponding $\tilde{\Omega}_{\mu}^{\prime}(\omega), \tilde{\Omega}_{Q, \mu}(\omega)$ elements. After the unitary transformation, the matrix equation (42) becomes diagonal, and equation (43) becomes antidiagonal. In order to have products with the same indices $\mu$, that is, $\tilde{\Omega}_{Q, \mu}(\omega) \cdot \tilde{\varphi}_{\mu}^{\prime}(\omega), \tilde{\Omega}_{Q, \mu}^{\prime}(\omega) \cdot \tilde{q}_{1}^{\prime}(\omega)\left(\right.$ instead of $\left.\tilde{\Omega}_{Q, 4}(\omega) \cdot \tilde{\varphi}_{1}^{\prime}(\omega), \tilde{\Omega}_{Q, 4}^{\prime}(\omega) \cdot \tilde{q}_{1}^{\prime}(\omega)\right)$ in the transformed equation (43), one should change the numbering of the $\tilde{\Omega}_{Q, \mu}(\omega), \tilde{\Omega}_{Q, \mu}^{\prime}(\omega)$ matrices eigenvalues to the opposite one $((1,2,3,4) \rightarrow(4,3,2,1))$. Then, when the parameters $\tilde{\varphi}_{\mu}^{\prime}(\omega), \tilde{q}_{\mu}^{\prime}(\omega)$ 
are found and substituted to the expression for $\tilde{\eta}_{\mu}^{\prime}(\omega)$ (diagonalized first equation of (40)), we obtain

$$
\begin{aligned}
&-\tilde{\bar{\eta}}_{\mu}^{\prime}(\omega)=\left[D_{\mu}(\omega) / B_{\mu}(\omega)-\beta \nu_{\mu}(\overrightarrow{0})\right]_{\mathrm{PGState}}^{-1}\left[2 / \tilde{\Omega}_{\mu}(\omega)-\left(1-\left\langle F^{(1)}(\omega)\right\rangle_{\mathrm{c}}\right)^{-1}-\beta \bar{\nu}_{\mu}(\overrightarrow{0})\right]^{-1} \\
& D_{\mu}(\omega)=\left[2\left\langle F^{(2)}(\omega)\right\rangle_{\mathrm{c}}-\tilde{\Omega}_{\mu}(\omega)\right]\left[2\left\langle F^{(4)}(\omega)\right\rangle_{\mathrm{c}}-{\tilde{\Omega^{\prime}}}_{Q, \mu}(\omega)\right] \\
&-\left[2\left\langle F^{(3)}(\omega)\right\rangle_{\mathrm{c}}-\tilde{\Omega}^{\prime}{ }_{\mu}(\omega)\right]\left[2\left\langle F^{(3)}(\omega)\right\rangle_{\mathrm{c}}-\tilde{\Omega}_{Q, \mu}(\omega)\right] \\
& B_{\mu}(\omega)=\left\langle F^{(2)}(\omega)\right\rangle_{\mathrm{c}} \cdot \tilde{\Omega}_{\mu}(\omega)\left[2\left\langle F^{(4)}(\omega)\right\rangle_{\mathrm{c}}-\tilde{\Omega}^{\prime}{ }_{Q, \mu}(\omega)\right] \\
&+\left\langle F^{(2)}(\omega)\right\rangle_{\mathrm{c}} \cdot \tilde{\Omega}_{Q, \mu}(\omega) \cdot{\tilde{\Omega^{\prime}}}_{\mu}(\omega)-2\left[\left\langle F^{(3)}(\omega)\right\rangle_{\mathrm{c}}\right]^{2} \tilde{\Omega}_{\mu}(\omega)
\end{aligned}
$$

In the case of ferroelectric ordering in $(50),(51)$ the eigenvalues of $\tilde{\Omega}_{\mu}(\omega), \tilde{\Omega}_{Q, \mu}(\omega), \tilde{\Omega}_{\mu}^{\prime}(\omega)$, $\tilde{\Omega}_{Q, \mu}^{\prime}(\omega)$ matrices are constructed on the matrix elements $\left\langle\Omega_{f f^{\prime}}(\omega)\right\rangle_{\mathrm{c}},\left\langle\Omega_{Q, f f^{\prime}}(\omega)\right\rangle_{\mathrm{c}},\left\langle\Omega_{f f^{\prime}}^{\prime}(\omega)\right\rangle_{\mathrm{c}}$ $\left\langle\Omega_{Q, f f^{\prime}}^{\prime}(\omega)\right\rangle_{\mathrm{c}}$, similarly to the matrix $\hat{\bar{\varphi}}^{\prime}(\omega)(48)$. In the case of antiferroelectric ordering, in these expressions the eigenvalues of $\tilde{\Omega}_{\mu}(\omega), \tilde{\Omega}_{Q, \mu}(\omega)$ matrices are constructed on the matrix elements $\left\langle\Omega_{f f^{\prime}}(\omega)\right\rangle_{\mathrm{c}} ;\left\langle\Omega_{Q, f f^{\prime}}(\omega)\right\rangle_{\mathrm{c}}$, similarly to the matrix $\hat{\bar{\varphi}}^{\prime}(\omega)(48)$, and the eigenvalues of the $\tilde{\Omega}_{\mu}^{\prime}(\omega)$, $\tilde{\Omega}_{Q, \mu}^{\prime}(\omega)$ matrices are constructed on the matrix elements $\left\langle\Omega_{f f^{\prime}}^{\prime}(\omega)\right\rangle_{\mathrm{c}} ;\left\langle\Omega_{Q, f f^{\prime}}^{\prime}(\omega)\right\rangle_{\mathrm{c}}$ similarly to the matrix $\hat{q}^{\prime}(\omega)(49)$. Let us note that in the case of a pure system the expression for $\tilde{\eta}_{\mu}^{\prime}(\omega)$ is analogous to (50) in the proton glass region, except that it does not contain the configurational averaging, and coincides with the expression given in [42].

In this work we shall explore temperature and composition dependences of the complex permittivity of the system

$$
\varepsilon_{a a}(\omega, T)=\varepsilon_{a a}^{0}+4 \pi \chi_{a a}(\omega, T), \quad \varepsilon_{a a}^{0}=1+4 \pi \chi_{a a}^{0}, \quad(a=1,2,3) .
$$

Dynamic susceptibility $\chi_{a a}(\omega, T)$ of the system is expressed via dynamic eigenvalues $\tilde{\eta}_{\mu}^{\prime}(\omega)(50)$ like in the static case (27) after the replacement $\tilde{\bar{\eta}}_{\mu}^{\prime} \rightarrow \tilde{\bar{\eta}}_{\mu}^{\prime}(\omega)$.

\section{Discussion}

In this section we analyze temperature dependencies of the Edwards-Anderson parameter $Q_{\mathrm{EA}}(T)$ and transverse $\varepsilon_{11}(\omega, T)$ permittivities for different compositions, as well as phase diagrams of $\mathrm{Rb}_{1-x}\left(\mathrm{ND}_{4}\right)_{x} \mathrm{D}_{2} \mathrm{PO}_{4}$ and $\mathrm{Rb}_{1-x}\left(\mathrm{NH}_{4}\right)_{x} \mathrm{H}_{2} \mathrm{AsO}_{4}$ compounds. It should be noted that the choice of the model parameters proposed below provides a good agreement with experimental data for specific heat, sublattice polarization, transverse and longitudinal permittivities for pure $\mathrm{ND}_{4} \mathrm{D}_{2} \mathrm{PO}_{4}$ and $\mathrm{RbH}_{2} \mathrm{AsO}_{4}, \mathrm{NH}_{4} \mathrm{H}_{2} \mathrm{AsO}_{4}$ [42] crystals in wide temperature and frequency ranges.

For the $\mathrm{Rb}_{1-x}\left(\mathrm{ND}_{4}\right)_{x} \mathrm{D}_{2} \mathrm{PO}_{4}$ mixture $\left(T_{\mathrm{N}}(x=1)=242 \mathrm{~K}\right)$ the set of the model parameters is in table 1 (we use everywhere $w_{1 \alpha}=\infty$ ). Dashes in table mean, that the given parameter is averaged over two states only (excluding the neutral state of the tetrahedron 0 (Glass)). We take into account the difference between $d_{\alpha}^{x}(G)$ (effective dipole moment of bond in proton glass phase) and $d_{\alpha}^{x}(F)$ (effective dipole moment of bond in phases with microscopic polarization).

It should be noted that crystal structure of $\mathrm{Rb}\left(\mathrm{H}_{1-y} \mathrm{D}_{y}\right)_{2} \mathrm{PO}_{4}$ is isomorphic to structure of $\mathrm{KH}_{2} \mathrm{PO}_{4}$ only at $y<0.90$. Thus, our results for crystal $\mathrm{RbD}_{2} \mathrm{PO}_{4}$ are purely theoretical and can differ from experimental data.

In figure 2 we show the temperature curves of the $Q_{\mathrm{EA}}(T)$ parameter for different compositions.

At $x=0.22$, the theoretical curve 3 well describes the experimental data of [15]. At the same time, at $x=0.44$, our results accord with the data of [14], but are lower than the values of [15]. We think that the reason for this is an incorrect determination of composition in [15]. 
Table 1. Parameters for the $\mathrm{Rb}_{1-x}\left(\mathrm{ND}_{4}\right)_{x} \mathrm{D}_{2} \mathrm{PO}_{4}$ compound.

\begin{tabular}{|l|l|l|l|l|l|}
\hline $\begin{array}{l}\text { Tetrahedron } \\
\text { state }\end{array}$ & $\varepsilon_{\alpha}$ & $w_{\alpha}$ & $\nu_{1, \alpha \alpha}(0)$ & $\nu_{2, \alpha \alpha}\left(k^{z}\right)$ & $\nu_{2, \alpha \alpha}(0)$ \\
$\mathrm{K}$ & $\mathrm{K}$ & $\mathrm{K}$ & $\mathrm{K}$ & $\mathrm{K}$ \\
\hline$+($ Ferro $)$ & 160 & 1100 & 22.76 & 25 & 20 \\
\hline 0 (Glass) & - & - & -44 & 40 & -60 \\
\hline- (Antiferro) & -140 & 750 & -40 & 67.44 & -20 \\
\hline
\end{tabular}

\begin{tabular}{|l|l|l|l|l|l|l|}
\hline $\begin{array}{l}\text { Tetrahedron } \\
\text { state }\end{array}$ & $\begin{array}{l}d_{\alpha}^{x}(G) 10^{-18} \\
\mathrm{esu} \cdot \mathrm{cm}\end{array}$ & $\begin{array}{l}d_{\alpha}^{x}(F) 10^{-18} \\
\mathrm{esu} \cdot \mathrm{cm}\end{array}$ & $\chi_{11}^{0}$ & $\begin{array}{l}v_{e} \\
10^{-21} \mathrm{~cm}\end{array}$ & $\begin{array}{l}\sqrt{Q_{G}}, \\
\mathrm{~K}\end{array}$ & $\begin{array}{l}\tau_{0, \alpha}^{x} \\
10^{-14}, \mathrm{~s}\end{array}$ \\
\hline$+($ Ferro $)$ & 6.5 & 6.5 & 0.8 & 0.209 & - & 3 \\
\hline 0 (Glass) & 7.1 & 2.0 & 0.7 & - & 24.5 & 6 \\
\hline$-($ Antiferro $)$ & 6.3 & 2.0 & 0.58 & 0.211 & - & 3 \\
\hline
\end{tabular}

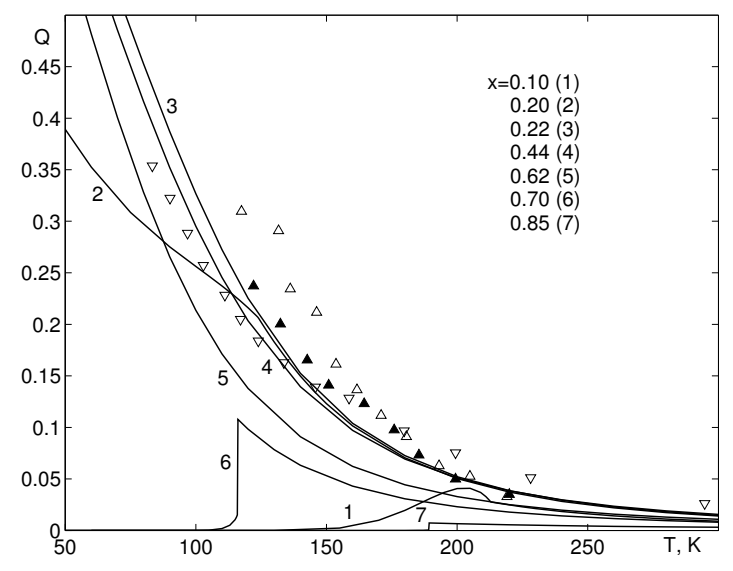

Figure 2. Temperature dependence of reduced Edwards-Anderson parameter $\mathrm{Q}_{\mathrm{EA}}$ of $\mathrm{Rb}_{1-x}\left(\mathrm{ND}_{4}\right)_{x} \mathrm{D}_{2} \mathrm{PO}_{4}$ compounds at different $x: 0.1-1 ; 0.2-2 ; 0.22-3, \boldsymbol{\Delta}[15] ; 0.44-4$, $\triangle[15], \nabla[14] ; 0.62-5 ; 0.7-6 ; 0.85-7$.

Figure 3 contains a comparison of calculations of the transverse static permittivity $\varepsilon_{11}(T)$ $\left(\varepsilon_{11}(T)=\varepsilon_{11}^{\prime}(\omega=0, T)\right)$ with experimental data for the real part of the permittivity $\varepsilon_{11}^{\prime}(\omega \neq 0, T)$ at low frequencies $\omega[18,16,2,43,44]$. In the cases of $x=0.24 ; 0.5$, the experimental points for $\varepsilon_{11}(T)$ from [18] are shown. A principal difference between static and dynamic results is observed in the proton glass region. While $\varepsilon_{11}(T)$ has a plateau at temperature lowering, $\varepsilon_{11}^{\prime}(\omega \neq 0, T)$ sharply falls to zero, the peak position shifting to higher temperatures with $\omega$ increasing. However, at temperatures above the peak, the dynamic and static permittivities practically coincide. This fact enables us to talk about qualitative agreement or disagreement between the theoretical curves for $\varepsilon_{11}(T)$ and experimental data for $\varepsilon_{11}^{\prime}(\omega \neq 0, T)$. For compositions $x=0.0$ (curve 1$) ; x=0.1$ (2); $x=0.2$ (3) the theoretical results only are shown. For $x=0.24(4), x=0.50$ (5), $x=0.62$ (6) the theoretical data correlate well with experiment. At $x=0.50$ the experimental values of $\varepsilon_{11}^{\prime}(\nu=10 \mathrm{kHz}, T)[16]$ are higher than the values of $\varepsilon_{11}(T)$ [18] even at high $T$. This could indicate an incorrect determination of the composition in one of these experiments. At $x=0.7(7)$ the calculated values are lower than experimental data of [18] for $1 \mathrm{kHz}$, while at $x=1(9)$ the calculated values are slightly higher than the experimental ones and than those calculated in [42] $\left(9^{\prime}\right)$. In the latter paper, a relation is used, which is a partial case of (22), (23) and (49), (50) at $x=0,1$, but a different set of the model parameters is used. Our corrected values of the model 
parameters permit us to describe thermodynamic and dielectric characteristics in the total range of compositions $x=[0,1]$. The worst agreement with experiment is observed at concentrations close to $x \sim 0.2$ and $x \sim 0.65$, which are the transition regions between the pure phases (ferroelectric ordering, glass, antiferroelectric ordering), which we do not take into account in this work.

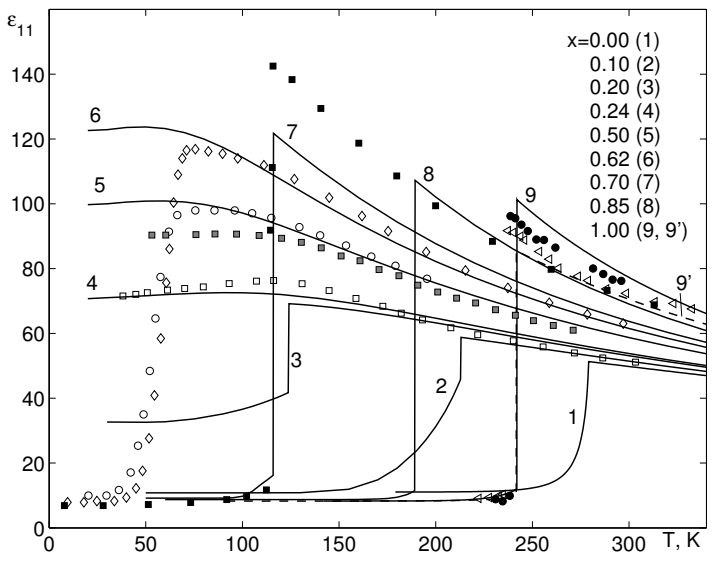

Figure 3. Temperature dependence of transverse dielectric permittivity $\varepsilon_{11}^{\prime}(2 \pi \nu, T)$ of $\mathrm{Rb}_{1-x}\left(\mathrm{ND}_{4}\right)_{x} \mathrm{D}_{2} \mathrm{PO}_{4}$ compounds at different $x: 0.0-1 ; 0.1-2 ; 0.2-3 ; 0.24-4$, 口 [18] (stat); $0.5-5$, [18] (stat), ○ [16] (10 kHz); $0.62-6, \diamond[2](300 \mathrm{~Hz}) ; 0.7-7$, - [18] $(1 \mathrm{kHz}) ; 0.85-8 ; 1.0-9,9^{\prime}[42], \bullet[43], \triangleleft[44]$. Solid lines are our calculations at $\nu=0$.

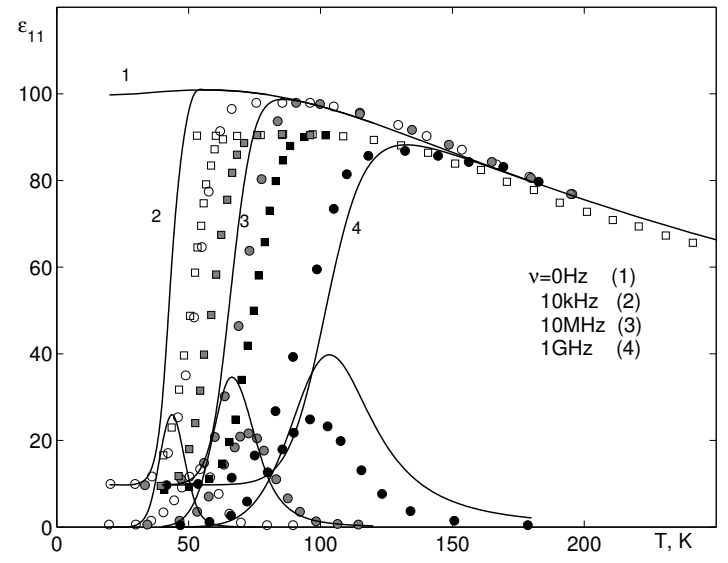

Figure 4. Temperature dependence of transverse dielectric permittivity $\varepsilon_{11}^{\prime}(2 \pi \nu, T)$ of $\mathrm{Rb}_{1-x}\left(\mathrm{ND}_{4}\right)_{x} \mathrm{D}_{2} \mathrm{PO}_{4}$ compounds for $x=0.5$ at different frequencies: $0 \mathrm{~Hz}-1: 1 \mathrm{~Hz}-\square$ [18]; $1 \mathrm{kHz}-$ [18]; $10 \mathrm{kHz}-2$, ○ [16]; $1 \mathrm{MHz}$ - - [18]; $10 \mathrm{MHz}-3$, ○ [16]; $1 \mathrm{GHz}$ $-4, \bullet[16]$.

Figure 4 illustrates the temperature dependences of the real $\varepsilon_{11}^{\prime}(\omega, T)$ and imaginary $\varepsilon_{11}^{\prime \prime}(\omega, T)$ parts of the permittivity at different frequencies for the proton-glass phase at $x=0.5$. The temperature peak of $\varepsilon_{11}^{\prime \prime}(\omega, T)$ corresponds to the inflection point of the $\varepsilon_{11}^{\prime}(\omega, T)$ curve. Calculations are performed at $\nu=0 \mathrm{~Hz}$ (curve 1); $10 \mathrm{kHz}(2) ; 10 \mathrm{MHz}(3) ; 1 \mathrm{GHz}$ (4). Experimental data for $\varepsilon_{11}^{\prime}(\omega, T)[18]$ at $\nu=1 \mathrm{~Hz} ; 10 \mathrm{kHz} ; 1 \mathrm{MHz}$ and for $\varepsilon_{11}^{\prime}(\omega, T), \varepsilon_{11}^{\prime \prime}(\omega, T)[16]$ at $\nu=10 \mathrm{kHz} ; 10 \mathrm{MHz}$; $1 \mathrm{GHz}$ are also shown. An essential dispersion of the presented experimental data should be noted. For $\varepsilon_{11}^{\prime}(\omega, T)$ the data of [16] in the region of low-temperature decrease approximately correspond to the data of [18] for essentially lower frequencies. The points for $\nu=10 \mathrm{kHz}[18]$ in the decrease region are shifted to higher temperatures as compared to the points for $\nu=10 \mathrm{kHz}[16]$. The latter are very close to the data for $\nu=1 \mathrm{~Hz}$ [18]. Then the points for $\nu=10 \mathrm{MHz} \varepsilon_{11}^{\prime}(\omega, T)[16]$ are shifted to lower temperatures as compared to the points for $\nu=1 \mathrm{MHz}$ [18]. Our theoretical curves for $\nu=10 \mathrm{kHz}$ (curve 2); $10 \mathrm{MHz}(3) ; 1 \mathrm{GHz}$ (4) qualitatively well describe the experimental temperature behavior of $\varepsilon_{11}^{\prime \prime}(\omega, T), \varepsilon_{11}^{\prime}(\omega, T)$ obtained in [16]. However, the theory yields a wider shift curves at frequency changing and a sharper and higher shape of the imaginary part.

We also calculated $\varepsilon_{11}^{\prime}(\omega, T), \varepsilon_{11}^{\prime \prime}(\omega, T)$ in the regions of ferroelectric and antiferroelectric ordering. At low frequencies the real parts of dynamic permittivity $\varepsilon_{11}^{\prime}(\omega, T)$ in the high-temperature range (near and above $T_{\mathrm{c}}, T_{\mathrm{N}}$ ) practically coincide with the static permittivities $\varepsilon_{11}(T)$. At low temperatures, however, $\varepsilon_{11}^{\prime \prime}(\omega, T)$ have peaks (inflection points of $\varepsilon_{11}^{\prime}(\omega, T)$ ), which correspond to the freezing line and indicate a possible occurrence of the proton glass phase at these values of $x$. On lowering $x$, positions of the low-temperature peak of $\varepsilon_{11}^{\prime \prime}(\omega, T)$ practically do not change, but their maximal values fastly decrease. We failed to find this peak numerically at $x<0.15$. A similar peak is detected in the antiferroelectric phase region at $0.65<x<0.70$. At the phase diagram, the freezing lines in the ferroelectric and antiferroelectric phases are continuations of the freezing lines of the proton glass region (figure 5 , dashed lines).

The phase diagram of $\mathrm{Rb}_{1-x}\left(\mathrm{ND}_{4}\right)_{x} \mathrm{D}_{2} \mathrm{PO}_{4}$ contains four regions. At high temperatures the system is in the ergodic proton glass state (EPG- ergodic proton phase). In this state the Edwards- 


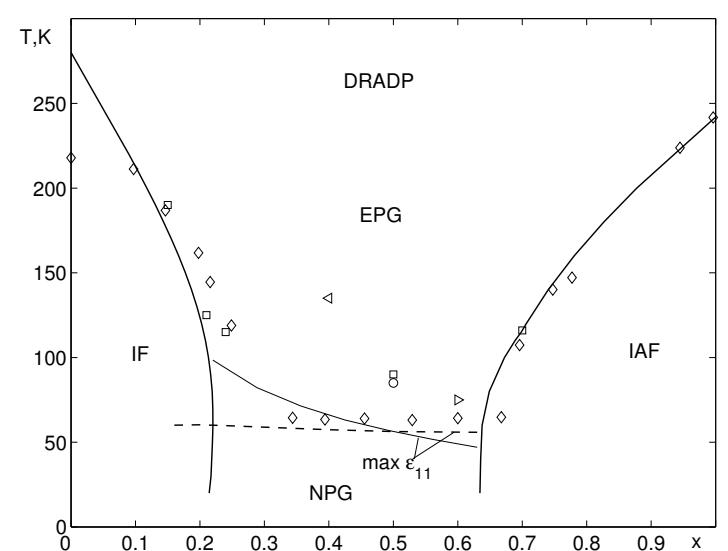

Figure 5. Phase diagram of $\mathrm{Rb}_{1-x}\left(\mathrm{ND}_{4}\right)_{x} \mathrm{D}_{2} \mathrm{PO}_{4} \quad$ compounds. $\diamond$ $[17], \square-[18], \circ-[16], \triangleright-[45], \triangleleft-[20]$.

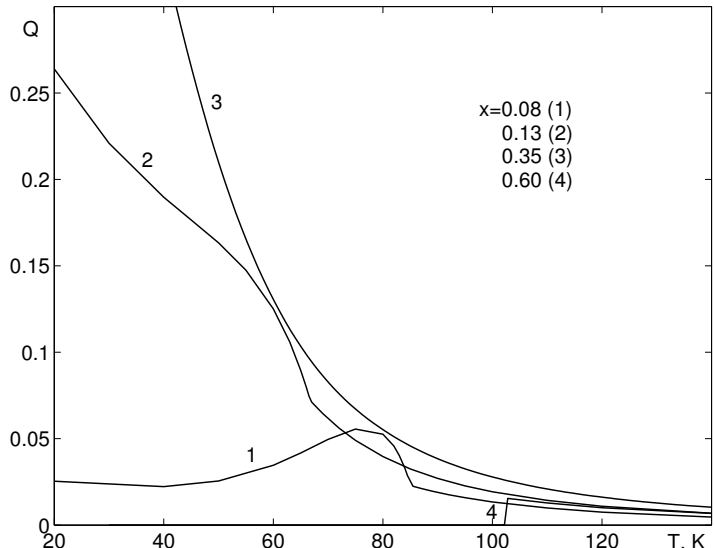

Figure 6. Temperature dependence of Edwards-Anderson parameter QEA of $\mathrm{Rb}_{1-x}\left(\mathrm{NH}_{4}\right)_{x} \mathrm{H}_{2} \mathrm{AsO}_{4}$ compounds at different $x: 0.08-1 ; 0.13-2 ; 0.35-3 ; 0.6-4$.

Anderson parameter is different from zero and decreases with temperature increasing. At $x<0.22$ and $x>0.65$ and at lowering temperature, the system goes to inhomogeneous ferroelectric (IFinhomogeneous ferroelectrics) or inhomogeneous antiferroelectric (IAF- inhomogeneous antiferroelectrics) states at $T_{\mathrm{c}}(x)$ and $T_{\mathrm{N}}(x)$, respectively (tetrahedron polarization appears at $T_{\mathrm{c}}(x)$, $T_{\mathrm{N}}(x)$ ). In the central region $0.22<x<0.65$ below the freezing lines $T_{g, 1}(x) \sim$ const (this temperature corresponds to the position of the low-temperature peak of $\left.\varepsilon_{11}^{\prime \prime}(\omega, T)\right)$ the system goes to the nonergodic proton glass state (NPG- nonergodic proton phase). This line continues in the regions $x<0.22$, where the proton glass state can coexist with ferroelectric ordering. Let us note that the experimentally observed regions of phase coexistence in some $\mathrm{Rb}_{1-x}\left(\mathrm{NH}_{4}\right)_{x} \mathrm{H}_{2} \mathrm{PO}_{4}$ type mixtures $\left(\mathrm{Rb}_{1-x}\left(\mathrm{NH}_{4}\right)_{x} \mathrm{H}_{2} \mathrm{AsO}_{4}, \mathrm{~K}_{1-x}\left(\mathrm{NH}_{4}\right)_{x} \mathrm{H}_{2} \mathrm{PO}_{4}\right)$ can be much wider, approaching the $x=0$, $x=1$ limits.

In $\mathrm{Rb}_{1-x}\left(\mathrm{NH}_{4}\right)_{x} \mathrm{H}_{2} \mathrm{AsO}_{4}$ mixtures $\left(T_{\mathrm{c}}(x=0)=110 \mathrm{~K}, T_{\mathrm{N}}(x=1)=216 \mathrm{~K}\right)$, an essential role in the low-temperature dynamics is played by the processes of proton tunneling. To describe them we need to go beyond the limits of Glauber kinetic equations. However, as we shall see, the proposed approach can be used for a qualitative description of dynamic phenomena in this compound.

The chosen set of the model parameters for $\mathrm{Rb}_{1-x}\left(\mathrm{NH}_{4}\right)_{x} \mathrm{H}_{2} \mathrm{AsO}_{4}$ is given in table 2 .

Table 2. Parameters for $\mathrm{Rb}_{1-x}\left(\mathrm{NH}_{4}\right)_{x} \mathrm{H}_{2} \mathrm{AsO}_{4}$.

\begin{tabular}{|l|l|l|l|l|l|}
\hline $\begin{array}{l}\text { Tetrahedron } \\
\text { state }\end{array}$ & $\varepsilon_{\alpha}$ & $w_{\alpha}$ & $\nu_{1, \alpha \alpha}(0)$ & $\nu_{2, \alpha \alpha}\left(k^{z}\right)$ & $\nu_{2, \alpha \alpha}(0)$ \\
$\mathrm{K}$ & $\mathrm{K}$ & $\mathrm{K}$ & $\mathrm{K}$ & $\mathrm{K}$ \\
\hline+ (Ferro) & 60 & 500 & 9.83 & 5 & 5 \\
\hline 0 (Glass) & - & - & -15 & 22 & -25 \\
\hline$-($ Antiferro $)$ & -100 & 470 & -80 & 75.19 & 5 \\
\hline
\end{tabular}

\begin{tabular}{|l|l|l|l|l|l|l|}
\hline $\begin{array}{l}\text { Tetrahedron } \\
\text { state }\end{array}$ & $\begin{array}{l}d_{\alpha}^{x}(G) 10^{-18} \\
\mathrm{esu} \cdot \mathrm{cm}\end{array}$ & $\begin{array}{l}d_{\alpha}^{x}(F) 10^{-18} \\
\mathrm{esu} \cdot \mathrm{cm}\end{array}$ & $\chi_{11}^{0}$ & $\begin{array}{l}v_{e} \\
10^{-21} \mathrm{~cm}\end{array}$ & $\begin{array}{l}\sqrt{Q_{G}}, \\
\mathrm{~K}\end{array}$ & $\begin{array}{l}\tau_{0, \alpha}^{x} \\
10^{-14}, \mathrm{~s}\end{array}$ \\
\hline$+($ Ferro $)$ & 5.1 & 5.1 & 0.7 & 0.2236 & - & 7 \\
\hline 0 (Glass) & 6.4 & 6.4 & 1.1 & - & 10 & 7 \\
\hline$-($ Antiferro $)$ & 6.3 & 2.0 & 0.7 & 0.2275 & - & 7 \\
\hline
\end{tabular}




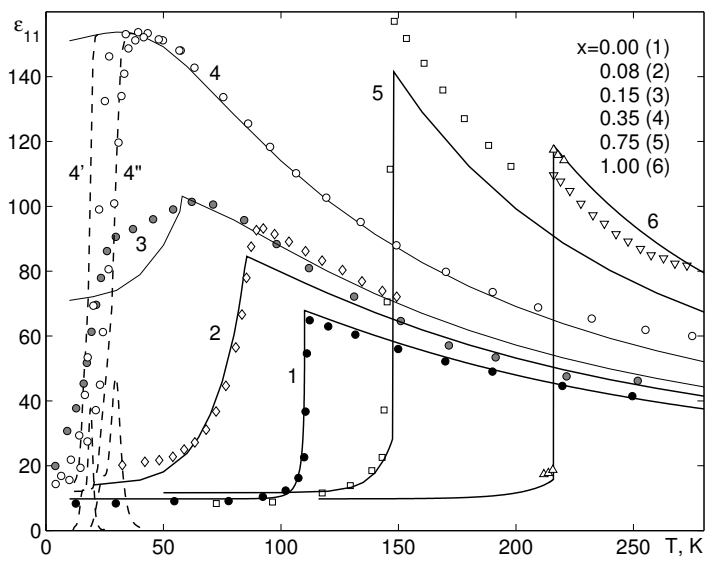

Figure 7. Temperature dependence of transverse dielectric permittivity $\varepsilon_{11}^{\prime}(2 \pi \nu, T)$ of $\mathrm{Rb}_{1-x}\left(\mathrm{NH}_{4}\right)_{x} \mathrm{H}_{2} \mathrm{AsO}_{4}$ compounds at different $x: 0.0-1, \bullet \quad[25] ; 0.08-2, \diamond \quad[29](1 \mathrm{kHz})$; $0.15-3$, ○ [25] $(1 \mathrm{~Hz}, 30 \mathrm{kHz}) ; 0.35-4,4^{\prime}, 4^{\prime \prime}$ (dashed lines), ○ [24] $(1 \mathrm{~Hz}, 30 \mathrm{kHz}) ; 0.75-5$, $[28](1 \mathrm{MHz}) ; 1.0-6, \nabla[47], \Delta$ [48]. Solid lines - our calculations at $\nu=0$.

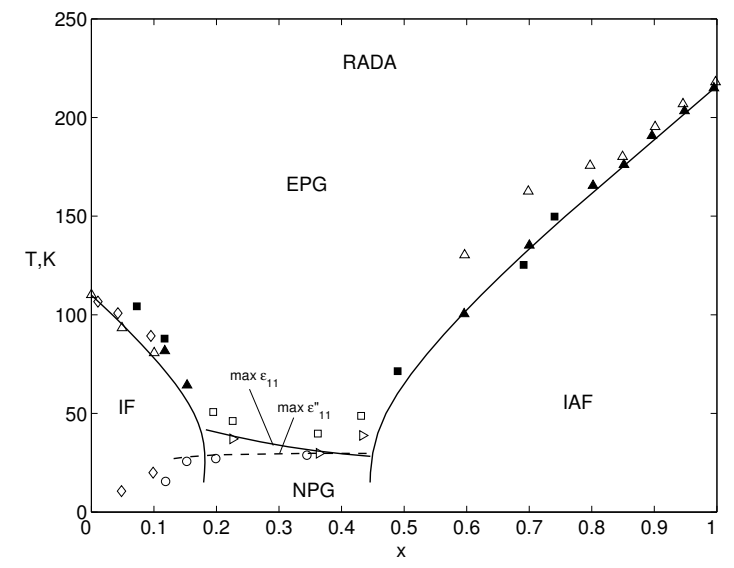

Figure 8. Phase diagram of $\mathrm{Rb}_{1-x}\left(\mathrm{NH}_{4}\right)_{x} \mathrm{H}_{2} \mathrm{AsO}_{4}$ compounds. $\diamond-[29]$, $\mathbf{}, \mathbf{-}, \triangleright-[28]$, ○$[26], \Delta, \boldsymbol{\Delta}-[23]$.

In figure 6 we plot the calculated temperature curves of the reduced Edwards-Anderson parameter $Q_{\mathrm{EA}}$ of $\mathrm{Rb}_{1-x}\left(\mathrm{NH}_{4}\right)_{x} \mathrm{H}_{2} \mathrm{AsO}_{4}$ at $x=0.08$ (curve 1); $x=0.13(2) ; x=0.35$ (3); $x=0.6$ (4). Unfortunately, no experimental data for this crystal are available.

In figure 7 we compare the calculated static transverse permittivity $\varepsilon_{11}(T)=\varepsilon_{11}^{\prime}(\omega=0, T)$ with experimental points for the real part of the transverse permittivity $\varepsilon_{11}^{\prime}(\omega \neq 0, T)$ at low frequencies $\omega$. The phase diagram is strongly asymmetric, and the proton glass state occurs in the region $x=[0.2 ; 0.45]$. As seen, a quantitative or qualitative agreement with experiment takes place in the regions far from the phase boundaries (curves 1,2 for the ferroelectric state and curves 5,6 for the antiferroelectric state). The value of $x=0.15$ corresponds to the intermediate phases between the ferroelectric state and the proton glass state. Below the permittivity peak, the theoretical curve deviates from the experimental points. For $x=0.35$ (approximately, a center of the proton glass phase) we compare the theoretical results with experimental data for $\nu=1 \mathrm{~Hz}, 30 \mathrm{kHz}$ of [25]. The theory yields a much faster decrease of $\varepsilon_{11}^{\prime}(\omega, T)$ with temperature and a very high and sharp curve of $\varepsilon_{11}^{\prime \prime}(\omega, T)$. The shape of this line for $\mathrm{Rb}_{1-x}\left(\mathrm{NH}_{4}\right)_{x} \mathrm{H}_{2} \mathrm{AsO}_{4}$ sharper than for $\mathrm{Rb}_{1-x}\left(\mathrm{ND}_{4}\right)_{x} \mathrm{D}_{2} \mathrm{PO}_{4}$ is due to an essential contribution of proton tunneling to the low-temperature dynamics. This effect 
is not taken into account by the proposed theory. However, we think that the present approach can be used to describe a position of the imaginary part of the permittivity (freezing line).

The phase diagram of $\mathrm{Rb}_{1-x}\left(\mathrm{NH}_{4}\right)_{x} \mathrm{H}_{2} \mathrm{AsO}_{4}$ (figure 8), like in the case of $\mathrm{Rb}_{1-x}\left(\mathrm{ND}_{4}\right)_{x} \mathrm{D}_{2} \mathrm{PO}_{4}$, contains four regions EPG, NPG, IF, IAF. The freezing line $T_{g, 1}(x)$ (dashed line) corresponds to the maximum of $\varepsilon_{11}^{\prime \prime}(\nu=1 \mathrm{~Hz}, T)$. According to experimental data $[29,26]$ this line is observed in the ferroelectric phase region down to $x=0.01$. With decreasing $x, T_{g, 1}(x) \rightarrow 0$. In calculations the decreasing line is observed down to $x \sim 0.15$. Overall, the calculated phase diagram correctly describes the experimental data, though some disagreement is observed. Thus, the used values of the model parameters yield a somewhat wider proton glass region $x \sim[0.18 ; 0.46]$ than the one experimentally observed $x \sim[0.22 ; 0.42]$. This disagreement can be due to different methods of identifying the proton glass state. Experimentally, X-ray scattering, NMR, and Raman scattering data are used to identify the phases.

\section{Conclusion}

In this work we propose a pseudospin model for the $\mathrm{Rb}_{1-x}\left(\mathrm{ND}_{4}\right)_{x} \mathrm{D}_{2} \mathrm{PO}_{4}$ type proton glasses, which takes into account the energy levels of hydrogens (deuterons) near $\mathrm{PO}_{4}$ groups, long-range interactions between hydrogen bonds, and an internal chaotic deformational field. Within the framework of the cluster approximation for short-range interactions and of the mean field approximation for long-range interactions, we obtain a system of equations for the state parameters (short-range cluster fields, long-range fields, and cluster field dispersion) and expressions for static longitudinal and transverse susceptibilities of glasses for the regions of ferroelectric, antiferroelectric, and proton glass states. Within the Glauber dynamics approach and cluster approximation we obtain a system of equations for time-dependent linear responses of polarization and proton glass order parameter. For the $\mathrm{Rb}_{1-x}\left(\mathrm{ND}_{4}\right)_{x} \mathrm{D}_{2} \mathrm{PO}_{4}$ mixture, the proposed theory satisfactorily describes a temperature behavior of real and imaginary parts of transverse permittivity at different frequencies in the "pure" phase regions $(x \sim 0.0 ; 0.5 ; 1.0)$. At the same time, the theory incorrectly describes the shape of the curve of an imaginary part of permittivity $\varepsilon_{11}^{\prime \prime}(T, \omega)$ for the $\mathrm{Rb}_{1-x}\left(\mathrm{NH}_{4}\right)_{x} \mathrm{H}_{2} \mathrm{AsO}_{4}$ mixture at low temperatures in the glass phase (the theoretical peak is too narrow and high). This can be due to neglecting the tunneling within the Glauber approach, which plays a crucial role in the dynamical processes in these compounds at low temperatures. The calculated freezing line slightly depends on concentration in the proton glass phase and continues down to $5 \%$ to the regions of ferroelectric ordering and antiferroelectric ordering. Hence, in our work the freezing line is determined in the same way as in experimental works. The phase diagrams for $\mathrm{Rb}_{1-x}\left(\mathrm{ND}_{4}\right)_{x} \mathrm{D}_{2} \mathrm{PO}_{4}, \mathrm{Rb}_{1-x}\left(\mathrm{NH}_{4}\right)_{x} \mathrm{H}_{2} \mathrm{AsO}_{4}$ obtained using dielectric calculations, are close to those constructed using experimental data.

\section{References}

1. Courtens E., J. Phys. (Paris) Lett., 1982, 43 L199.

2. Courtens E., Phys. Rev. B, 1986, 33, No. 4, 2975.

3. Moriya K., Matsuo T., Suga H., Terauchi H., Jpn. J. Appl. Phys., 1985, 24 Suppl. 24-2, 955.

4. Takashige M., Terauchi H., Miura Y., Hoshino S., Nakamura T., Jpn. J. Appl. Phys., 1985, 24 Suppl. $24-2,947$.

5. Takashige M., Terauchi H., Miura Y., Hoshino S., J. Phys. Soc. Jpn., 1985, 54, No. 9, 3250.

6. Terauchi H., Ferroelectrics, 1985, 64, 87.

7. Courtens E., Jpn. J. Appl. Phys, 1985, 24, 70.

8. Hattori T., Araki H., Nakashima S., Mitsuishi A., Terauchi H., J. Phys. Soc. Jpn., 1988, 57, No. 3, 1127.

9. Hayase S., Sakashita H., Terauchi H., Ferroelectrics, 1987, 72, 245.

10. Nagata T., Iwata M., Orihara H., Ishibashi Y., Miura Y., Mamiya T., Terauchi H., J. Phys. Soc. Jpn., 1997, 66, No. 5, 1503.

11. Choi Y.S., Kim J.J., Eur.Lett., 2004, 65, No. 1, 55-60.

12. Dolinsek J., Arcon D., Zalar B., Pirc R. and Blinc R., Phys.Rev.B, 1996, 54, No. 10, R6811. 
13. Blinc R., Dolinsek J., Ailion D.C., Gunther D., Zumer S., Phys. Rev. B, 1986, 57, No. 22, 2826.

14. Blinc R., Dolinsek J., Pirc R., Tadic B., Zalar B., Phys. Rev. Lett., 1989, 63, No. 20, 2248.

15. Chen S., Ailion D.C., Phys.Rev.B, 1990, 42, No. 10, 5945.

16. He P., J. Phys. Soc. Jpn., 1991, 60, No. 1, 313.

17. Korner N., Pfammatter Ch., Kind R., Phys. Rev. Lett., 1993, 70, No. 9, 1283.

18. Kutnjak Z., Pirc R., Levstik A., Levstik I., Filipic C., Blinc R., Phys.Rev., 1994, 50, No. 17, 12421.

19. Kim B.G., Kim J.J., Phys.Rev.B, 1997, 55, No. 9, 5558.

20. Ko J.H., Kim B.G., Kim J.J., Fujimori H., Miyajima S., J. Phys.: Condens. Matter., 1995, $14,4403$.

21. Gregorovic A., Zalar B., Blinc R., Ailion D.C., Phys.Rev.B, 1999, 60, No. 1, 76.

22. Banys J., Kajokas A., Lapinskas S., Brillingas A., Grigas J., Petzelt J., Kamba S., J. Phys. B, 2002, 14, 3725 .

23. Trybula Z, Stankowski J, Szczepanska L., Blinc R., Weiss Al., Dalal N.S., Ferroelectrics, 1988, 79, 335.

24. Trybula Z., Schmidt V.H., Drumheller J.E., He D., Li Z., Phys. Rev.B, 1989, 40, No. 7, 5289.

25. Trybula Z., Schmidt V.H., Drumheller J.E., Phys. Rev.B, 1991, 43, No. 1, 1287.

26. Trybula Z, Stankowski J., Condens. Matter Phys., 1998, 1, No. 3(15), 311.

27. Trybula Z. Kaszynski J., Ferroelectrics, 2004, 298, 347.

28. Kim S., Kwun S., Phys. Rev. B, 1990, 42, No. 1, 638.

29. Pinto J., Schmidt V.H., Ferroelectrics, 1993, 141, 207.

30. Prelovcek P., Blinc R., J. Phys. C.: Solid State Phys., 1982, 15, L985.

31. Matsushita E., Matsubara T., Prog. Theor. Phys., 1984, 71, No. 2, 235.

32. Matsushita E., Matsubara T., J. Phys. Soc. Jpn., 1985, 54, No. 3, 1161.

33. Pirc R., Tadic B., Blinc R., Phys. Rev. B, 1987, 36, No. 16, 8607.

34. Cevc P., Zalar B., Blinc R., Solid State Comm., 1989, 70, No. 4, 451.

35. Kind R., Blinc R., Dolinsek J., Korner N., Zalar B., Cevc P., Dalal N., DeLooze J., Phys. Rev. B, 1991, 43, No. 4, 2511.

36. Banerjee V., Dattagupta S., Phys. Rev B, 2003, 68, 054202.

37. Korynevskii N.A., Solovyan V.B., Ferroelectrics, 2005, 317, 19.

38. Korynevskii N.A., Solovyan V.B., Phase Transition, 2007, 80, No. 1-2, 55.

39. Levitskii R.R., Korinevskii N.A., Stasyuk I.V., Ukr. Phys. J., 1974, 19, No. 8, 1288 (in Russian).

40. Levitskii R.R., Sorokov S.I., Vdovych A.S., Ferroelectrics, 2005, 316, 111.

41. Sorokov S.I., Levitskii R.R., Vdovych A.S., Condens. Matter Phys., 2005, 8, No. 3(43), 603.

42. Levitsky R.R., Zachek I.R., Vdovych A.S. Preprint of the Institute for Condensed Matter Physics, ICMP-08-04U, Lviv, 2008 (in Ukrainian).

43. Volkova E.N., Izrailenko A.N., Kristallografiya, 1983, 28, No. 6, 1217 (in Russian).

44. Mason W.P., Mattias B.T., Phys. Rev., 1952, 88, No. 3, 477.

45. Levstik A., Filipic C., Kutnjak Z., Levstik I., Pirc R., Tadic B., Blinc R., Phys. Rev. Lett., 1991, 66, No. $18,2368$.

46. Fairall C.W., Reese W., Phys. Rev. B. 1974, 10, No. 3, 882.

47. Berdowski J., Opilski A., J. Crystal Growth, 1978, 43, 381.

48. Gesi K., Ozawa K., J. Phys. Soc. Jpn., 1984. 53, No. 12, 4405. 


\title{
Термодинаміка та комплексна діелектрична проникність змішаних кристалів типу $\mathrm{Rb}_{1-x}\left(\mathrm{NH}_{4}\right)_{x} \mathrm{H}_{2} \mathrm{PO}_{4}$
}

\author{
Р.Р.Левицький ${ }^{1}$, С.І.Сороков ${ }^{1}$, Я.Станковскі ${ }^{2}$, 3.Трибула ${ }^{2}$, А.С.Вдович ${ }^{1}$ \\ 1 Інститут фізики конденсованих систем НАН України, Україна, Львів 79011, вул. Свєнціцького 1 \\ 2 Інститут молекулярної фізики НАН,Польща, Познань 60-179, вул. Смолуховського 17
}

Отримано 10 червня 2008 р.

\begin{abstract}
Запропонована псевдоспінова модель протонних стекол типу $\mathrm{Rb}_{1-x}\left(\mathrm{NH}_{4}\right)_{x} \mathrm{H}_{2} \mathrm{PO}_{4}\left(\mathrm{Rb}_{1-x}\left(\mathrm{ND}_{4}\right)_{x}\right.$ $\mathrm{D}_{2} \mathrm{PO}_{4}$ ), яка враховує енергетичні рівні протонів (дейтронів) біля тетраедра $\mathrm{PO}_{4}$, далекосяжну взаємодію між водневими зв'язками і внутрішнє хаотичне деформаційне поле. В рамках кластерного наближення і наближення середнього поля по далекодіїі виведена система рівнянь для параметрів стану для областей, які знаходяться в феро-, антифероелектричному станах, і в стані протонного скла. В рамках глауберівської динаміки виведена система рівнянь для частотнозалежних лінійних відгуків полярізації і параметра протонного скла. Отримано якісний опис температурної поведінки діелектричних проникностей сполук $\mathrm{K}_{1-x}\left(\mathrm{ND}_{4}\right)_{x} \mathrm{D}_{2} \mathrm{PO}_{4}$ i $\mathrm{Rb}_{1-x}\left(\mathrm{NH}_{4}\right)_{x} \mathrm{H}_{2} \mathrm{AsO}_{4}$ при різних частотах. Обговорюються причини низькотемпературного піку уявної частини діелектричних проникностей в протонних стеклах.
\end{abstract}

Ключові слова: протонне скло, кластерне наближення, глауберівська динаміка

PACS: $75.10 . H k, 75.10 . \mathrm{Nr}, 77.22 . \mathrm{Ch}, 77.22 . \mathrm{Gm}, 77.84 . \mathrm{Fa}$ 